Y. Teranishi

Nagoya Math. J.

Vol. 117 (1990), 173-205

\title{
UNIVERSAL INDUCED CHARACTERS AND RESTRICTION RULES FOR THE CLASSICAL GROUPS
}

\author{
YASUO TERANISHI \\ Dedicated to Professor H. Morikawa on his sixtieth birthday
}

\section{Introduction}

The purpose of this paper is the study of some basic properties of universal induced characters and their applications to the representation theory of the classical groups (for the definition of a universal induced character, see $\S 3$ ).

The starting point was the paper [F] by E. Formanek on matrix invariants. In his paper [F], Formanek has investigated the Hilbert series for the ring of matrix invariants from the point of view of the representation theory of the general linear group and the symmetric group. In this paper we shall study polynomial concomitants of a group from the same point of view.

The induced character of a representation is very useful and fundamental tool in the representation theory. Let $G$ be a subgroup of the general linear group $G L_{n}$ over the complex number field $C$ and $\rho$ a finite dimensional representation of $G$. We shall generalize the notion of the induced character $\chi\left(\rho \uparrow{ }_{G}^{G 1}\right)$ and introduce the universal induced character $\chi\left(\rho \uparrow_{G}^{G L_{n}}\right)$. The universal induced character is defined as an element of the symmetric formal power series ring in infinitely many variables.

One of the most fundamental properties of induced representations is the Frobenius reciprocity theorem. So our first object in section 3 is to establish a result for universal induced characters analogous to the Frobenius reciprocity theorem. This is done by Theorem 3.1 in this paper. In the proof of Theorem 3.1, we use the classical Molien-Weyl theorem, which gives an expression for the Hilbert series of a graded module of polynomial concomitants as an integral over a maximal torus of a compact Lie group.

Received January 17, 1989. 
In our consideration, close relations. with classical invariant theory appear. For example, let $V$ be an $n$-dimensional vector space and $G$ a subgroup of $G L(V)$. Consider the diagonal action of $G$ on the vector space $\oplus V$, the direct sum of countably infinitely many copies of $V$. Then denoting by $\prod N$ the countably infinitely many copies of $N$, the ring $C[\oplus V]^{G}$ of polynomial invariants is a $\prod N$-graded ring over $C$ in a canonical way and the Hilbert series

$$
\begin{gathered}
H\left(C[\oplus V]^{G}, t_{1}, t_{2}, \cdots\right)=\sum_{\underline{\underline{G}} \in \mathbb{N}} \operatorname{dim}_{C} C[\oplus V]_{\underline{d}}^{G} t_{1}^{d_{1}} t_{2}^{d_{2}} \cdots, \\
\text { with } \quad \underline{d}=\left(d_{1}, d_{2}, \cdots\right)
\end{gathered}
$$

of the $\prod N$-graded ring $C[\oplus V]^{G}$ is equal to the universal induced character $\chi\left(1 \uparrow_{G}^{G L_{n}}\right)$ for the trivial representation 1 of $G$.

By using the (generalized) Frobenius reciprocity theorem (Theorem 3.1), we shall prove some basic properties of universal induced characters.

Let $G$ be one of the classical groups of type $B_{n}, C_{n}$ and $\mathrm{D}_{n}$ :

$$
G= \begin{cases}S O(2 n+1) & \text { for type } \mathrm{B}_{n} \\ S p(2 n) & \text { for type } \mathrm{C}_{n} \\ S O(2 n) & \text { fro type } \mathrm{D}_{n} .\end{cases}
$$

Roughly speaking the irreducible representations of $G$ are parametrized by the partitions of length $\leq n$. For a partition $\lambda$ of length $\leq n$, let $\rho_{G}(\lambda)$ denote the irreducible representation corresponding to $\lambda$. Under the natural embedding $G \subset G L_{\nu}(\nu=2 n$, if $G=S p(2 n)$ or $S O(2 n)$, and $\nu=2 n+1$, if $G=S O(2 n+1)$ ), we present a combinatorial expression for the universal induced character $\chi\left(\rho_{G}(\lambda) \uparrow_{G}^{G L_{\nu}}\right)$.

Let $\rho$ be an irreducible representation of the general linear group $G L_{\nu}$ and consider the restriction $\rho \downarrow_{G}^{G L_{\nu}}$ of the representation:

$$
\rho \downarrow_{G}^{G L_{\nu}}=\sum_{\lambda} m_{\lambda,} \rho_{G}(\lambda)
$$

H. Weyl ([W-2], Chap. VII) has calculated the multiplicities $m_{\lambda}$. Since the irreducible representations of the classical groups are parametrized by the partitions, the multiplicities $m_{\lambda}$ should be calculated from partitions only, R.C. King ([K-1], [K-2]) and K. Koike-I. Terada [K-T] have presented the algorithm to calculate the multiplicities $m_{\lambda}$ from given partitions. The expression for the universal induced character presents the combinatorial algorithm to calculate the multiplicities. 
This paper is organized as follows. In rsection 1, we study polynomial concomitants of a group $G$ ( $G$-equivariant polynomial mappings). In section 2, we review some basic results on the representations of the general linear group $G L_{n}$ and the symmetric group $S_{n}$ which we will use later.

In section 3 , we introduce the notion of a universal induced character and study its fundamental properties. In section 4 , we review the results on the representations of the classical groups of type $\mathrm{B}_{n}, \mathrm{C}_{n}$ and $\mathrm{D}_{n}$, which we will use in section 5 . In section 5 , we study the universal induced characters of the irreducible representations of the classical groups, and prove the restriction rules for the classical groups.

This research has been done while the author was visiting The University of Mannheim of West Germany. He wishes to express his hearty thanks to Professor H. Popp for an enjoyable stay at The University of Mannheim.

\section{§1. Concomitant}

Let $G$ be a linearly reductive group acting on finite dimensional vector spaces $V$ and $W$ over a field $k$ of characteristic zero. Let $k[V, W]$ (resp. $k(V, W)$ ) denote the set of all polynomial (resp. rational) mappings $V \rightarrow W$. Then the group $G$ acts on $k[V, W]$ (resp. $k(V, W)$ ) as follows: for $f \in k[V, W]$ (resp. $k(V, W)$ and $g \in G, g \cdot f(x)=g f\left(g^{-1} x\right)$.

A polynomial (resp. rational) mapping $f: V \rightarrow W$ is called a polynomial (resp. rational) concomitant of $G$ if it is invariant under the action of $G$ on $k[V, W]$ (resp. $k(V, W)$ ). Equivalently for all $x \in V$ and $g \in G, g f(x)=f(g \cdot x)$. We denote by $k[V, W]^{G}\left(\operatorname{resp} . k(V, W)^{G}\right)$ the $k$-linear space of polynomial (resp. rational) concomitants of $G$.

Suppose $V_{i}, 1 \leq i \leq r$, be a family of finite dimensional $k$-vector spaces on which $G$ acts linearly. Then $G$ acts on $\oplus_{1 \leq i \leq r} V_{i}$, the direct sum of $V_{i}$, by $g\left(x_{1}, \cdots, x_{r}\right)=\left(g x_{1}, \cdots, g x_{r}\right)$, for $g \in G$ and $\left(x_{1}, \cdots, x_{r}\right)$ $\oplus_{1 \leq i \leq r} V_{i}$. If $G$ acts linearly on a vector space $W$, a polynomial (resp. rational) mapping $f: \oplus_{1 \leq i \leq r} V_{i} \rightarrow W$ is called a simultaneous polynomial (resp. rational) concomitant if $f$ satisfies

$$
g\left(f\left(x_{1}, \cdots, x_{r}\right)\right)=f\left(g\left(x_{1}, \cdots, x_{r}\right)\right),
$$

for all $g \in G$ and $\left(x_{1}, \cdots, x_{r}\right) \in \oplus_{1 \leq i \leq r} V_{i}$.

Let $k[V]$ (resp. $k(V)$ ) be the ring of polynomial (resp. rational) functions on $V$. We denote the ring (resp. field) of polynomial (resp. rational) 
invariants on $V$ by $k[V]\left(\right.$ resp. $\left.k(V)^{G}\right)$. Then $k[V, W]^{G}$ is a $k[V]^{G}$-module by

$$
(h \cdot f)(x):=h(x) f(x), \quad \text { for } \quad h \in k[V]^{G} \text { and } f \in k[V, W]^{G} .
$$

Similarly $k(V, W)^{G}$ is a vector space over the field $k(V)^{G}$. Moreover $k[V, W]^{G}$ is a finitely generated $k[V]^{G}$-module and

$$
k(V, W)^{a}=k(V)^{G} \cdot k[V, W]^{G} .
$$

A fundamental theorem of Hochster and Roberts [H-R, Main Theorem] says that the ring $k[V]^{G}$ is a Cohen-Macaulay ring. A similar result for $k[V, W]^{G}$ is also true if $G$ is a finite group. For the sake of reader's convenience, we give a proof (cf. [S] 3.2 Theorem).

Proposition 1.1. If $G$ is finite group, $k[V, W]^{G}$ is a Cohen-Macaulay module over $k[V]^{G}$.

Proof. Let $\left(\theta_{1}, \cdots, \theta_{n}\right)$ be a homogeneous system of parameters of $k[V]^{G}$. Since $G$ is a finite group, $k[V]$ is integral over the subring $k[V]^{G}$ and hence $\left(\theta_{1}, \cdots, \theta_{n}\right)$ is a homogeneous system of parameters of $k[V]$. Trivially, $k[V, W]$ is a Cohen-Macaulay module over $k[V]$. Therefore $k[V, W]$ is a free module over the subring $k\left[\theta_{1}, \cdots, \theta_{n}\right]$ of $k[V]$ generated by elements $\theta_{1}, \cdots, \theta_{n}$, and hence $k[V, W] / \theta_{1} k[V, W]+\cdots+\theta_{n} k[V, W]$ is a finite dimensional vector space over $k$. Consider the projection operator $\natural:=|G|^{-1} \sum_{g \in G} g: k[V, W] \rightarrow k[V, W]^{G}$. Then $q$ bives a decomposition

$$
k[V, W]=k[V, W]^{G} \oplus M, \quad M=\left\{f \in k[V, W], f^{\natural}=0\right\}
$$

as $k[V]^{a}$-module. We choose a basis $\left(\bar{f}_{1}, \cdots, \bar{f}_{k}\right)$ of the $k$-vector space $k[V, W] / \theta_{1} k[V, W]+\cdots+\theta_{n} k[V, W]$ such that $\bar{f}_{1}, \cdots, \bar{f}_{m}$ is a basis for $k[V, W]^{G} / \theta_{1} k[V, W]^{G}+\cdots+\theta_{n} k[V, W]^{G}$ and $\bar{f}_{m+1}, \cdots, \bar{f}_{k}$ is a basis for $M / \theta_{1} M+\cdots+\theta_{n} M$. Let $f_{1}, \cdots, f_{m}$ be representatives in $k[V, W]^{a}$ for $\bar{f}_{1}, \cdots, \bar{f}_{m}$, respectively. This gives a decomposition

$$
k[V, W]^{G}=\oplus_{1 \leq i \leq m} f_{i} k\left[\theta_{1}, \cdots, \theta_{n}\right],
$$

which completes the proof.

Let $e_{1}, \cdots, e_{n}$ be a basis for $V$ and $f_{1}, \cdots, f_{m}$ a basis for $W$. A polynomial mapping $u: V \rightarrow W$,

$$
u\left(\sum_{j} x_{j} e_{j}\right)=\sum_{i} u_{i}\left(x_{1}, \cdots, x_{n}\right) f_{i}
$$

has degree $d$ if all polynomials $u_{i}\left(x_{1}, \cdots, x_{n}\right)$ are homogeneous polynomials of degree $d$. 
For every $d \in N$, let $k[V, W]_{d}^{G}$ denote the $k$-vector space of polynomial concomitants of degree $d$. Then the $k$-module $k[V, W]^{G}$ of polynomial concomitants is an $N$-graded $k$-module

$$
k[V, W]^{G}=\oplus_{d \in N} k[V, W]_{d}^{G} .
$$

Similarly, let $\left(G, \rho_{i}, V_{i}\right), 1 \leq i \leq r,(G, r, W)$ be finite dimensional representations of $G$. For each $i \in\{1,2, \cdots, r\}$, let $e(i)_{1}, \cdots, e(i)_{n_{i}}$ be a basis of $V_{i},\left(n_{i}=\operatorname{dim}_{k} V_{i}\right)$. A polynomial mapping $u: \oplus_{1 \leq i \leq r} V_{i} \rightarrow W$,

$$
u\left(\sum_{j} x(1)_{j} e(1)_{j}, \cdots, \sum_{j} x(r)_{j} e(r)_{j}\right)=\sum_{k} u_{k}\left(x(i)_{j} \mid 1 \leq i \leq r, 1 \leq j \leq d_{i}\right) f_{k}
$$

has degree $\underline{d}=\left(d_{1}, \cdots, d_{r}\right) \in N_{r}$, if for each $i \in\{1,2, \cdots, r\}$, all the polynomials

$$
u_{k}\left(x(i)_{j} \mid 1 \leq j \leq n_{i}\right)
$$

are homogeneous with respect to the variables

$$
x(i)_{1}, \cdots, x(i)_{n_{i}}
$$

of degree $d_{i}$.

For every $\underline{d}=\left(d_{1}, \cdots, d_{r}\right) \in N^{r}$, let $k\left[\oplus_{1 \leq i \leq m} V, W\right]_{d}^{G}$ be the $k$-vector space of simultaneous polynomial concomitants of degree $\underline{d}$. Then $k\left[\oplus_{1 \leq i \leq r} V_{i}, W\right]^{G}$ is an $N^{r}$-graded module:

$$
k\left[\oplus_{1 \leq i \leq r} V_{i}, W\right]^{G}=\oplus_{d \in N^{r}} k\left[\oplus_{1 \leq i \leq r} V_{i}, W\right]_{d}^{G} .
$$

In general, for an $N^{r}$-graded $k$-module $M$, the associated Hilbert series $H(M, \underline{t}), \underline{t}=\left(t_{1}, \cdots, t_{r}\right)$, is defined to be

$$
H(M, \underline{t})=\sum_{\underline{d} \in N^{r}} \operatorname{dim}_{k} \cdot M_{\underline{d}} \underline{t}^{\underline{d}},
$$

where

$$
\underline{t}^{\underline{d}}=t_{1}^{d_{1}} \cdots t_{r}^{d_{r}} .
$$

Hereafter, throughout this paper, we take the complex number field $C$ as the basic field $k$. If $(G, \rho, V)$ and $(G, \gamma, W)$ are finite dimensional continuous complex representations of a compact group $G$ with the normalized Haar measure $d g$, then the Molien-Weyl formula gives an expression for the Hilbert series $H\left(C[V, W]^{G}, t\right)$ as an integral over the group $G$ :

$$
H\left([V, W]^{G}, t\right)=\int_{G} \frac{\operatorname{Tr}\left(\gamma(g)^{-1}\right.}{\operatorname{det}\left(1_{n}-t \rho(g)\right)} d g \quad\left(n=\operatorname{dim}_{c} V\right) .
$$


Similarly for finite dimensional complex representations $\left(G, \rho_{i}, V_{i}\right), 1 \leq$ $i \leq r$, and $(G, \gamma, W)$ of a compact group $G$, we obtain:

Proposition 1.2 (Molien-Weyl formula).

$$
H\left(\boldsymbol{C}\left[\oplus_{1 \leq i \leq r} V_{i}, W\right]^{G}, \underline{t}\right)=\int_{G} \frac{\operatorname{Tr}\left(\gamma(g)^{-1}\right)}{\prod_{1 \leq i \leq r} \operatorname{det}\left(1_{n_{i}}-t_{i} \rho_{i}(g)\right.} d g,
$$

where

$$
n_{i}=\operatorname{dim}_{C} V_{i} \text { for } 1 \leq i \leq r, \quad \text { and } \quad \underline{t}=\left(t_{1}, \cdots, t_{r}\right) .
$$

For a compact group $G$, let $T$ be a maximal torus of $G, d g$ the normalized Haar-measure on $T$ and $W$ the Weyl group of $G$. Let $\left\{\alpha_{1}, \alpha_{2}, \cdots\right.$, $\alpha_{N}$ \} be the set of roots of $G$ with respect to $T$ and set

$$
d \Omega=\prod_{1 \leq i \leq N}\left(1-\alpha_{i}\right) d g .
$$

Let $G$ be a compact subgroup of $G L(V)$ and $(G, \rho, W)$ a finite dimensional representation of $G$. Then by Weyl's integration formula, we have

$$
H\left(C\left[\oplus^{r} V, W\right]^{G}, \underline{t}\right)=\frac{1}{|W|} \int_{G} \frac{\operatorname{Tr}\left(\rho(g)^{-1}\right)}{\prod_{1 \leq i \leq r} \operatorname{det}\left(1_{n}-t_{i} g\right)} d \Omega .
$$

Proposition 1.3. Let $G$ be a semi-simple linear algebraic group and $V$ a finite dimensional representation of $G$. Suppose that $V$ is G-equivalent as a G-module to its dual space $V^{*}$. Then for any $r>1$ and $\underline{d}=$ $\left(d_{1}, \cdots, d_{r}\right) \in N^{r}$,

$$
\operatorname{dim}_{C} C\left[\oplus^{r} V, V\right]_{\underline{d}}^{G}=\operatorname{dim}_{C} C\left[\oplus^{r+1} V\right]_{(\underline{d}, 1)}^{G},
$$

Proof. By Weyl's unitarian trick we may assume that $G$ is a compact group. Let $\rho$ denote the representation of $G$ on $V$. Since $V=V^{*}$ as $G$-modules, $\operatorname{Tr}(\rho(g))=\operatorname{Tr}\left(\rho(g)^{-1}\right)$. Notice that

$$
\operatorname{det}\left(1_{n}-t x\right)^{-1}=1+\operatorname{Tr}(x) t+\cdots,
$$

for any $n$ by $n$ matrix $X$ and a variable $t$. Then using the Molien-Weyl formula, we obtain the desired result.

As an application of the proposition above, we prove the following

Proposition 1.4. Let $G$ be a semi-simple connected linear algebraic group and Lie $(G)$ its Lie algebra on which $G$ acts as the adjoint representation. Let (, ) denote the Killing form on Lie $(G) \times \operatorname{Lie}(G)$. For $x=$ $\left(x_{1}, \cdots, x_{r}, x_{r+1}\right) \in \oplus^{r+1} \operatorname{Lie}(G)$, set $x^{\prime}=\left(x_{1}, \cdots, x_{r}\right)$ and $x^{\prime \prime}=x_{r+1}$. Then for any $\underline{d} \in N^{r}$, the $C$-linear map 
$T: C\left[\oplus^{r} \operatorname{Lie}(G), \operatorname{Lie}(G)\right]_{\underline{d}}^{G} \longrightarrow C\left[\oplus^{r+1} \operatorname{Lie}(G)\right]_{(\underline{d}, 1)}^{G}$

defined by

$$
T(f)(x)=\left(f\left(x^{\prime}\right), x^{\prime \prime}\right), \quad f \in C\left[\oplus^{r} \operatorname{Lie}(G), \operatorname{Lie}(G)\right]_{\underline{d}}^{G} \quad \text { and } \quad x \in \oplus^{r+1} \operatorname{Lie}(G)
$$

is an isomorphism between $\boldsymbol{C}$-vector spaces $\boldsymbol{C}\left[\oplus^{r} \operatorname{Lie}(G) \text {, Lie }(G)\right]_{d}^{G}$ and $C\left[\oplus^{r+1} \operatorname{Lie}(G)\right]_{(\underline{d}, 1)}^{G}$.

Proof. Since $G$ is semi-simple, the Killing form (,) is $G$-invariant non-degenerate bilinear form and hence the map $T$ is well-defined and injective. By the previous proposition, $T$ is surjective.

Using the same method as in the proof of 4.3 Theorem [T], we obtain

Proposition 1.5. Let $G$ be a semi-simple connected linear algebraic group. Then for any $r>3$, the Hilbert series $R(\underline{t})$ for $C\left[\oplus^{r} \operatorname{Lie}(G)\right.$, Lie $(G)]^{G}$ satisfies the functional equation

$$
R\left(t_{1}^{-1}, \cdots, t_{r}^{-1}\right)=(-1)^{d}\left(t_{1} \cdots t_{r}\right)^{\operatorname{dim} G} R\left(t_{1}, \cdots, t_{r}\right),
$$

where

$$
d=(r-1) \operatorname{dim} G .
$$

\section{§2. Representations of the general linear groups and symmetric groups}

In this section we review definitions, notations and some results on the representations of the general groups and symmetric groups. We use the terminology of Macdonald [M] although we occasionally modify it.

A finite or infinite sequence

$$
\lambda=\left(\lambda_{1}, \lambda_{2}, \cdots\right)
$$

of nonnegative integers in decreasing order $\lambda_{1} \geq \lambda_{2} \geq \cdots$ and containing only finitely many nonzero terms is called a partition. For a partition $\lambda$, the associated Young diagram (denoted also by $\lambda$ ) is the set of points

$$
\left\{(i, j) \mid i, j \in Z, 1 \leq i, 1 \leq j \leq \lambda_{i}\right\} \in Z^{2} .
$$

If $(i, j) \in \boldsymbol{Z}^{2}$ is contained in a Young diagram $\lambda$, then $(i, j)$ is called a node of $\lambda$. The $i$-th row (resp. column) of a Young diagram $\lambda$ consists of nodes whose first (resp. second) coordinates are $i$. For example if $\lambda=$ $(4,3,2,1)$, the associated Young diagram is 


$$
\lambda=\begin{array}{llll}
\times & \times & \times & \times \\
\times & \times & \times & \\
\times & \times & & \\
\times & & &
\end{array}
$$

For a partition $\lambda$, the number of nonzero terms in $\lambda$ is called the length of $\lambda$ and will be denoted by $\ell(\lambda)$. The size of $\lambda$ is defined to be the sum of all terms in $\lambda$ and will be denoted by $|\lambda|$ :

$$
|\lambda|=\lambda_{1}+\lambda_{2}+\cdots .
$$

We denote by ${ }^{t} \lambda$ the conjugate of a partition $\lambda$.

Consider the graded ring $Z\left[t_{1}, \cdots, t_{n}\right]$ of polynomials in $n$ indeterminates $t_{1}, \cdots, t_{n}$ with integer coefficients and let $\Lambda_{n}$ denote the graded ring of symmetric polynomials in $n$ variables:

$$
\Lambda_{n}=Z\left[t_{1}, \cdots, t_{n}\right]^{s_{n}},
$$

where $S_{n}$ denotes the symmetric group on $n$ letters which acts on $Z\left[t_{1}, \cdots, t_{n}\right]$ by permuting the variables.

For each $\underline{d}=\left(d_{1}, \cdots, d_{n}\right) \in N^{n}$ define

$$
\underline{t}^{\underline{d}}=t_{1}^{d_{1}} \cdots t_{n}^{d_{n}},
$$

and for each partition $\lambda$ of length $\leq n$, the monomial symmetric polynomial $m_{\lambda}$ is defined by

$$
m_{\lambda}=\sum \underline{t}^{\underline{d}},
$$

where the sum is over all distinct permutations $d$ of $\lambda_{1}, \cdots, \lambda_{n}$. Then the monomial symmetric polynomials $m$ 's form a $Z$-basis of $\Lambda_{n}$. Let $\pi_{m, n}$ : $\Lambda_{m} \rightarrow \Lambda_{n}(m>n)$ be the homomorphism of graded rings defined by $\pi_{m, n}\left(t_{i}\right)$ $=t_{i}$ if $1 \leq i \leq n$ and $\pi_{m, n}\left(t_{i}\right)=0$ if $n<i$. Then the $\left(\Lambda_{m}, \pi_{m, n}\right)$ satisfy a compatibility condition of a projective system. We denote the projective limit of this system in the category of graded rings by $\Lambda$, so that $\Lambda=$ $\varliminf \Lambda_{n}$. We call this projective limit the ring of symmetric functions in infinitely many variables.

For any partition $\lambda$ of length $\leq n$, setting

$$
\begin{aligned}
a_{\lambda} & =a_{\lambda}\left(t_{1}, \cdots, t_{n}\right) \\
& =\sum_{\sigma \in S_{n}}(\operatorname{sign} \sigma) t_{\sigma(1)}^{\lambda_{1}} \cdots t_{\sigma(n)}^{\lambda_{n}},
\end{aligned}
$$

the Schur polynomial $s_{\lambda}=s_{\lambda}\left(t_{1}, \cdots, t_{n}\right)$ is defined by 


$$
s_{\lambda}=a_{\lambda+\delta} / a_{\delta},
$$

where

$$
\delta=(n-1, n-2, \cdots, 1,0) .
$$

Then the Schur polynomials $s_{\lambda}$ corresponding to the partitions of length $\leq n$ form a $Z$-basis for $\Lambda_{n}$. Since $s_{\lambda}\left(t_{1}, \cdots, t_{n}\right)=0$ if $\ell(\lambda)>n$ and $\pi_{m, n}\left(s_{\lambda}\left(t_{1}, \cdots, t_{m}\right)\right)=s_{\lambda}\left(t_{1}, \cdots, t_{n}\right)$, if $m \geq n, s_{\lambda}\left(t_{1}, \cdots, t_{n}\right), n \in N$, form a projective system and hence we may define a symmetric functions $s_{\lambda}$ in infinitely many variables. The canonical projections $\pi_{n}: \Lambda \rightarrow \Lambda_{n}$ carry $s_{\lambda}$ to $s_{\lambda}\left(t_{1}, \cdots, t_{n}\right)$. The symmetric function $s_{\lambda}$ is called the Schur function corresponding to $\lambda$. The set $\left\{s_{\lambda} \mid \lambda\right.$ a partition $\}$ forms a $Z$-linear basis for 1. Hence for two partitions $\lambda$ and $\mu, s_{\lambda} s_{\mu}$ is a $Z$-linear combination of Schur functions:

$$
s_{\lambda} s_{\mu}=\sum_{\nu} L R_{\lambda \mu}^{\nu} s_{\nu}, \quad L R_{\lambda \mu}^{\nu} \in Z
$$

The coefficients $L R_{\lambda \mu}^{\nu}$ are called the Littlewood-Richardson coefficients. The Littlewood-Richardson rule gives a combinatorial description for computing $L R_{\lambda \mu}^{\nu}$. Similarly for partitions $\lambda_{1}, \cdots, \lambda_{r}$, the (general) Littlewood-Richardson coefficients $L R_{\lambda_{1}, \ldots, \lambda_{r}}^{\lambda}$ are defined by

$$
s_{\lambda_{1}} \cdots s_{\lambda_{r}}=\sum_{\lambda_{r}} L R_{\lambda_{1}, \cdots, \lambda_{r}}^{\lambda} s_{\lambda},
$$

where the sum is over all partitions $\lambda$. If $r=1$, we employ the convention

$$
L R_{\lambda}^{\nu}= \begin{cases}1 & \text { if } \lambda=\nu, \\ 0 & \text { otherwise }\end{cases}
$$

We denote by $\operatorname{Mod}\left(G L_{n}\right)$ the Grothendieck ring of finite dimensional polynomial $G L_{n}$-modules. For each finite dimensional $G L$-module $M$, let $\chi(M)=\chi(M)\left(t_{1}, \cdots, t_{n}\right)$ denote its $G L_{n}$-character. Then $\chi(M)$ is a symmetric polynomial in $t_{1}, \cdots, t_{n}$ and $M$ is irreducible if and only if $\chi(M)=$ $s_{\lambda}\left(t_{1}, \cdots, t_{n}\right)$ for some partition $\lambda$ of length $\leq n$. Thus the map $M \rightarrow \chi(M)$ induces a ring isomorphism between $\operatorname{Mod}\left(G L_{n}\right)$ and $\Lambda_{n}$.

The equivalence classes of finite dimensional irreducible polynomial $G L_{n}$-modules correspond to bijectively to the partitions of length $\leq n$. Hence hereafter, if $M$ is a finite dimensional irreducible $G L_{n}$-module corresponding to a partition $\lambda$ of length $\leq n$, we shall write it by the same symbol $\lambda$. 
Let $\operatorname{Mod}\left(S_{n}\right)$ denote the Grothendieck ring of finite dimensional $S_{n^{-}}$ modules. For each finite dimensional $S_{n}$-module $[M]$, consider $\operatorname{ch}[M]$, where ch stands for the characteristic map (see $[\mathrm{M}]$, p. 82). Then ch $[M]$ is a symmetric polynomial of degree $n$ and $M$ is irreducible if and only if $\operatorname{ch}[M]=s_{\lambda}$, the Schur function associated with a partition of size $n$. Hence the map $[M] \rightarrow \operatorname{ch}[M]$ induces a bijective correspondence between the isomorphism classes of finite dimensional irreducible $S_{n}$-module and the partitions of size $n$. So, if $[M]$ is a finite dimensional irreducible $S_{n}$ module corresponding to a partitions $\lambda$ of size $n$, we shall write it by [ $\lambda]$.

For two finite dimensional $S$-modules $[M]$ and $[N]$, consider the diagonal action of $S_{n}$ on the tensor product $[M] \otimes[N]$. The map

$$
\operatorname{Mod}\left(S_{n}\right) \otimes \operatorname{Mod}\left(S_{n}\right) \longrightarrow \operatorname{Mod}\left(S_{n}\right)
$$

induced by this action of $S_{n}$ is called the inner product. Let $\Lambda^{n}$ be the degree $n$ part of $\Lambda$ and define the inner product on it by

$$
\operatorname{ch}([M] \otimes[N])=\operatorname{ch}[M] * \operatorname{ch}[N] .
$$

In particular if $\lambda$ and $\mu$ are partitions of size $n$, we have

$$
s_{\lambda} * s_{\mu}=\operatorname{ch}([\lambda] \otimes[\mu]) .
$$

Similarly, for partitions $\lambda_{1}, \cdots, \lambda_{r}$ of size $n$, we define $s_{\lambda_{1}} * \cdots * s_{\lambda_{r}}$ by

$$
s_{\lambda_{1}} * \cdots * s_{\lambda_{r}}=\operatorname{ch}\left(\left[\lambda_{1}\right] \otimes \cdots \otimes\left[\lambda_{r}\right]\right) .
$$

For a partition $\lambda$ of size $n$, we denote by $\chi^{\lambda}$ the associated irreducible character of $S_{n}$. The following proposition was proved by I. Schur [(S], p. 68) for $r=2$ and his proof works as well for general case. For reader's convenience, the proof is here included.

Proposition 2.1. Consider $r, r \geq 2$, infinite sequences of independent variables:

$$
x=\left(x_{1}, x_{2}, \cdots\right), \quad y=\left(y_{1}, y_{2}, \cdots\right), \cdots, \quad z=\left(z_{1}, z_{2}, \cdots\right)
$$

and take an infinite sequence $t=\left(t_{1}, t_{2}, \cdots\right)$ of independent variables. Then

$$
\begin{aligned}
& \prod_{i, j, \cdots, k, m \in N_{+}}\left(1-x_{i} y_{j} \cdots z_{k} t_{m}\right)^{-1} \\
& \quad=\sum\left(s_{\lambda} * s_{\mu} * \cdots * s_{\nu}\right)(t) s_{\lambda}(x) s_{\mu}(y) \cdots s_{\nu}(z),
\end{aligned}
$$

where the sum is over all partitions $\lambda, \mu, \cdots, \nu$ with $|\lambda|=|\mu|=, \cdots,=|\nu|$.

Proof. We set $R=\prod_{i, j, \cdots, k, m \in N_{+}}\left(1-x_{i} y_{j} \cdots z_{k} t_{m}\right)^{-1}$. Then clearly $R$ is a symmetric function in $x, y, \cdots, z$ and $t$. Hence $R$ has the form 


$$
R=\sum_{\alpha} f_{\lambda \mu \ldots \nu}^{\alpha} s_{\lambda}(x) s_{\mu}(y) \cdots s_{\nu}(z) s_{\alpha}(t) \quad \text { with } \quad f_{\lambda \mu \ldots \nu}^{\alpha} \in Z
$$

On the other hand, one has

$$
\begin{aligned}
R= & \sum_{\kappa_{1}, \kappa_{2}}, \cdots \frac{1}{\kappa_{1} ! \kappa_{2} ! \cdots}\left(\frac{s_{1}(x) s_{1}(y) \cdots s_{1}(z) s_{1}(t)}{1}\right)^{\kappa_{1}} \\
& \times\left(\frac{s_{2}(x) s_{2}(y) \cdots s_{2}(z) s_{2}(t)}{2}\right)^{\kappa_{2}} \cdots
\end{aligned}
$$

where $\kappa_{1}, \kappa_{2}$, are over all nonnegative integers, and $s_{i}, i \in N_{+}$, stand for the power sums of the variables:

$$
s_{i}(x):=x_{1}^{i}+x_{2}^{i}+\cdots .
$$

Recall the Frobenius character formula for the symmetric group:

$$
s_{1}^{\kappa_{1}}(t) s_{2}^{\kappa_{2}}(t) \cdots=\sum_{\lambda} \chi^{\lambda}\left(\kappa_{1}, \kappa_{2}, \cdots\right) s_{\lambda}(t),
$$

where $\left(\kappa_{1}, \kappa_{2}, \cdots\right)$ is a permutation with cycle length $\kappa_{1}, \kappa_{2}, \cdots, \chi^{\lambda}\left(\kappa_{1}, \kappa_{2}, \cdots\right)$ stands for the value of the character of the symmetric group corresponding to a partition $\lambda$, evaluated on $\left(\kappa_{1}, \kappa_{2}, \ldots\right)$ and the sum is over all partitions of size $\kappa_{1}+\kappa_{2}+\cdots$.

Using the Frobenius character formula, we get

$$
\begin{aligned}
R= & \sum_{\kappa_{1}, \kappa_{2}, \ldots} \frac{1}{\kappa_{1} ! \kappa_{2} ! \cdots} \sum_{\lambda, \mu, \cdots, \nu} \chi^{\lambda}\left(\kappa_{1}, \kappa_{2}, \cdots\right) \chi^{\mu}\left(\kappa_{1}, \kappa_{2}, \cdots\right) \cdots \\
& \times \chi^{\nu}\left(\kappa_{1}, \kappa_{2}, \cdots\right) s_{\lambda}(x) s_{\mu}(y) \cdots s_{\nu}(z)\left(\frac{s_{1}(t)}{1}\right)^{\kappa_{1}}\left(\frac{s_{2}(t)}{2}\right)^{\kappa_{2}} \cdots
\end{aligned}
$$

Since for each partition $\alpha$,

$$
s_{\alpha}(t)=\sum_{\kappa_{1}, \kappa_{2}, \ldots} \frac{\chi^{\alpha}\left(\kappa_{1}, \kappa_{2}, \cdots\right)}{\kappa_{1} ! \kappa_{2} ! \cdots}\left(\frac{s_{1}(t)}{1}\right)^{\kappa_{1}}\left(\frac{s_{2}(t)}{2}\right)^{\kappa_{2}} \cdots
$$

we obtain

$$
\chi^{\lambda}\left(\kappa_{1}, \kappa_{2}, \cdots\right) \chi^{\mu}\left(\kappa_{1}, \kappa_{2}, \cdots\right) \cdots \chi^{\nu}\left(\kappa_{1}, \kappa_{2}, \cdots\right)=\sum_{\alpha} f_{\lambda \mu \cdots \nu}^{\alpha} \chi^{\nu}\left(\kappa_{1}, \kappa_{2}, \cdots\right),
$$

which completes the proof.

Let $M$ be a finite dimensional irreducible polynomial $G L_{r}$-module corresponding to a partition $\lambda$ of size $r$ and consider the sub-vector space [M] of $M$ defined by

$$
[M]:=\left\{f \begin{array}{l}
f \in M \text { and } D\left(t_{1}, \cdots, t_{r}\right)=f\left(t_{1} \cdots t_{r}\right) f \\
\text { for all diagonal matrices } D\left(t_{1}, \cdots, t_{r}\right) \\
\text { whose main diagonal entries are } t_{1}, \cdots, t_{r}
\end{array}\right\} .
$$


Then identifying the symmetric group $S_{r}$ with the permutation matrices in $G L_{r},[M]$ is an $S_{r}$-module. The following proposition will be used later:

Proposition 2.2 ([G], §6). With notation as above, $[M]$ is a finite dimensional irreducible $S_{r}$-module corresponding to the partition $\lambda$.

We can regard a non-negative integer $k$ as a partition of length 1 (denoted by $(k)$ ). For two partitions $\lambda=\left(\lambda_{1}, \lambda_{2}, \cdots\right)$ and $\mu=\left(\mu_{1}, \mu_{2}, \cdots\right)$, let $K_{\lambda, \mu}$ denote the number of Young tableaux of shape $\lambda$ and weight $\mu$. Then it follows easily from the Littlewood-Richardson rule that $L R_{\left(\mu_{1}\right),\left(\mu_{2}\right)}^{\lambda}, \ldots$ $=K_{\lambda, \mu}$.

\section{§3. Universal induced characters}

Let $D(r)$ denote the subgroup of $G L_{r}$ consisting of diagonal matrices and let $D\left(t_{1}, \cdots, t_{r}\right)$ denote the diagonal matrix whose main diagonal entries are $t_{1}, \cdots, t_{r}$. Let $M$ be an $N^{r}$-graded polynomial $G L_{r}$-module over $C$ and suppose that, for each $\underline{d}=\left(d_{1}, \cdots, d_{r}\right) \in N^{r}$, the $C$-vector space $M_{\underline{d}}$ is finite dimensional and is given by

$$
M_{\underline{d}}=\left\{\begin{array}{ll}
f \in M & \begin{array}{l}
D\left(t_{1}, \cdots, t_{r}\right) f=t_{1}^{d_{1}} \cdots t_{r}^{d_{r}} f, \quad \text { for all } \\
D\left(t_{1}, \cdots, t_{r}\right) \in D(r)
\end{array}
\end{array}\right\} .
$$

Then, for each $d \in N$, the $C$-vector space $M_{d}$ spanned by all homogeneous elements of total degree $d$ is a finite dimensional $G L$-representation space and its character $\chi\left(M_{d}\right)$ is equal to $\sum \operatorname{dim}_{c} M_{d} t^{\underline{d}}$, where the sum is over all $\underline{d}=\left(d_{1}, \cdots, d_{r}\right) \in N^{r}$ with $|\underline{d}|=d$ and $t^{\underline{d}}=t_{1}^{d_{1}} \cdots t_{r}^{d_{r}}$.

The character $\chi(M)$ of $M$ is defined to be

$$
\begin{aligned}
\chi(M) & =\sum_{d \in N} \chi\left(M_{d}\right) \\
& =\sum_{\underline{d} \in N^{r}} \operatorname{dim}_{C} M_{\underline{d}} t^{\underline{\underline{d}}} \\
& =H(M, \underline{t}) .
\end{aligned}
$$

The character of $M$ is an element of the ring of formal power series $\boldsymbol{Z} \llbracket t_{1}, \cdots, t_{r} \rrbracket$ and equal to the Hilbert series of $M$.

Let $V$ be an $n$-dimensional complex vector space, $G$ a linearly reductive subgroup of $G L_{n}(=G L(V))$ and $(G, \rho, W)$ a finite dimensional complex representation of $G$ on a vector space $W$. From now on, for each $r \in N$, we denote by $\rho \uparrow_{G}^{G L_{n}}$ the $C$ vector space $C\left[\oplus^{r} V, W\right]^{a}$ of simultaneous polynomial concomitants $f: V \oplus \cdots \oplus V(r$ copies of $V) \rightarrow W$. 
We have seen that $\rho \uparrow_{G}^{G L n}(r)$ is an $N^{r}$-graded, $G L_{r}$-module and the character of it is equal to the Hilbert series of $\rho \uparrow{ }_{G}^{G L_{n}}(r)$. It is clear that $\chi\left(\rho \uparrow{ }_{G}^{G L_{n}}(r)\right)$ is a symmetric function in $t_{1}, \cdots, t_{r}$.

Let $t_{1}, \cdots, t_{r}$ be commuting indeterminates and consider the ring of formal power series $Z \llbracket t_{1}, \cdots, t_{r} \rrbracket$ with $Z$-coefficients. Let $\Lambda_{r}$ denote the subring of $Z \llbracket t_{1}, \cdots, t_{r} \rrbracket$ of symmetric functions in $t_{1}, \cdots, t_{r} . \quad \Lambda_{r}$ is an $N^{r}$. graded ring whose grading is inherited from $Z \llbracket t_{1}, \cdots, t_{r} \rrbracket$. Suppose that $r>s$. Then

$$
\pi_{r, s}\left(t_{i}\right)= \begin{cases}t_{i}, & 1 \leq i \leq s \\ 0, & s+1 \leq i \leq r\end{cases}
$$

defines a degree preserving surjection

$$
\pi_{r, s}: \Lambda_{r} \longrightarrow \Lambda_{s} \text {. }
$$

The system $\left\{\Lambda_{r}, r \in N\right\}$ satisfies the compatibility condition of a projective system in the category of commutative rings. We set

$$
\Lambda:=\varliminf^{i m},
$$

and call it the ring of symmetric formal power series in infinitely many variables $t_{1}, t_{2}, \cdots$.

If $r>s$, then

$$
\pi_{r, s}\left(\chi\left(\rho \uparrow{ }_{G}^{G L_{n}}(r)\right)\left(t_{1}, \cdots, t_{r}\right)=\chi\left(\rho \uparrow_{G}^{G L_{n}}(s)\right)\left(t_{1}, \cdots, t_{s}\right),\right.
$$

and hence there is a well-defined element $\chi\left(\rho \uparrow_{G}^{G L_{n}}\right)$ of $\Lambda$ such that the canonical projection $\pi_{r}: \Lambda \rightarrow \Lambda_{r}$ carry $\chi\left(\rho \uparrow{ }_{G}^{G L_{n}}\right)$ to $\chi\left(\rho \uparrow{ }_{G}^{G L_{n}}(r)\right)$ for all $r \in N$. We call $\chi\left(\rho \uparrow{ }_{G}^{G L_{n}}\right)$ the universal induced character of the representation $(G, \rho, W)$. Notice that $\rho \uparrow_{G}^{G L_{n}}(n)$ is the (usual) induced representation of $(G, \rho, W)$ and $\chi\left(\rho \uparrow_{G}^{G L_{n}}(n)\right)$ is the induced character.

Since the Schur functions are $Z$-linear basis of $\Lambda$, there are integers $c_{\lambda}$ such that

$$
\chi\left(\rho \uparrow{ }_{G}^{G L_{n}}\right)=\sum c_{\lambda} s_{\lambda},
$$

where $\lambda$ varies over all partitions. For each $\lambda$, the integer $c_{\lambda}$ is called the multiplicity of $\lambda$ in $\chi\left(\rho \uparrow_{G}^{G L_{n}}\right)$ and will be denoted by $m\left(\rho \uparrow_{G}^{G L_{n}}, \lambda\right)$. For a partition $\lambda$ with $\ell(\lambda) \leq n$, let $\lambda \uparrow_{G}^{G L_{n}}$ denote the restriction of the irreducible representation of $G L_{n}$ corresponding to $\lambda$.

\subsection{The Frobenius reciprocity theorem}

In this section, we prove the following 
Theorem 3.1 (The Frobenius reciprocity theorem for the universal induced characters). Let $(G, \rho, W)$ be a finite dimensional irreducible representation of a compact subgroup $G$ of $G L_{n}$. Then, for each partition $\lambda$,

$$
m\left(\rho \uparrow{ }_{G}^{G L_{n}}, \lambda\right)= \begin{cases}m\left(\lambda \downarrow_{G}^{G L_{n}}, \rho\right), & \text { if } \ell(\lambda) \leq n, \\ 0, & \text { otherwise },\end{cases}
$$

where $m\left(\lambda \downarrow_{G}^{G L_{n}}, \rho\right)$ denotes the multiplicity of $\rho$ in $\lambda \downarrow_{G}^{G L_{n}}$. In other words,

$$
\chi\left(\rho \uparrow{ }_{G}^{G L_{n}}\right)=\sum_{\lambda} m\left(\lambda \downarrow_{G}^{G L_{n}}, \rho\right) s_{\lambda},
$$

where the sum is over all partitions of length $\leq n$.

Proof. Let $x=\left(x_{1}, x_{2}, \cdots\right)$ and $y=\left(y_{1}, y_{2}, \cdots\right)$ be two finite or infinite sequences of independent variables. Then, by Cauchy's formula,

$$
\prod_{i, j}\left(1-x_{i} y_{j}\right)^{-1}=\sum_{\lambda} s_{\lambda}(x) s_{\lambda}(y),
$$

where the sum is over all partitions. Let $T$ be a maximal torus of $G$. Then we may assume that $T$ has of the form

$$
T=\left\{\left(u^{\alpha_{1}}, \cdots, u^{\alpha_{n}}\right) \in D(n)\right\},
$$

where

$$
u^{\alpha_{i}}=\varepsilon_{1}^{\alpha_{i 1}} \cdots \varepsilon_{r}^{\alpha_{i r}}, \quad \text { with } \quad\left(\alpha_{i 1}, \cdots, \alpha_{i r}\right) \in Z,
$$

$r=\operatorname{rank}$ of $G$, for $1 \leq i \leq n$.

Let $r$ be a non-negative integer and set

$$
x_{i}=\left\{\begin{array}{ll}
u^{\alpha_{i}}, & 1 \leq i \leq n \\
0, & n<i
\end{array} \text { and } y_{j}= \begin{cases}t_{j}, & 1 \leq j \leq r \\
0, & r<j\end{cases}\right.
$$

Then we obtain

$$
\prod_{i, j}\left(1-u^{\alpha_{i}} t_{j}\right)^{-1}=\sum s_{\lambda}\left(u^{\alpha_{i}}: 1 \leq i \leq n\right) s_{\lambda}\left(t_{1}, \cdots, t_{r}\right) .
$$

On the other hand,

$$
s_{\lambda}\left(u^{\alpha_{i}}: 1 \leq i \leq n\right)= \begin{cases}\chi\left(\lambda \downarrow_{G}^{G L_{n}}\right)\left(\varepsilon_{1}, \cdots, \varepsilon_{n}\right), & \text { if } \ell(\lambda) \leq n \\ 0, & \text { if } \ell(\lambda)>n\end{cases}
$$

and, if $\ell(\lambda) \leq n$,

$$
\frac{1}{|W|} \int_{T} \chi\left(\lambda \downarrow_{G}^{G L_{n}}\right) \chi(\rho(g))^{-1} d \Omega=m\left(\lambda \downarrow_{G}^{G L_{n}}, \rho\right) .
$$

Hence, by the Molien-Weyl formula, we have 


$$
\begin{aligned}
\chi\left(\rho \uparrow{ }_{G}^{G L_{n}}(r)\right) & =\frac{1}{|W|} \int_{T} \frac{\operatorname{Tr}(\rho(g))^{-1}}{\prod_{1 \leq i \leq r} \operatorname{det}\left(1_{n}-g t_{i}\right)} d \Omega \\
& =\frac{1}{|W|} \int_{T} \frac{\operatorname{Tr}(\rho(g))^{-1}}{\prod_{\substack{1 \leq i \leq n \\
1 \leq j \leq r}}\left(1-u^{\alpha_{i}} t_{j}\right)} d \Omega \\
& =\sum_{\ell(\lambda) \leq n} m\left(\lambda \downarrow_{G}^{G L_{n}}, \rho\right) s_{\lambda}\left(t_{1}, \cdots, t_{r}\right),
\end{aligned}
$$

which completes the proof.

If $r=n$, the theorem above gives the usual Frobenius reciprocity theorem for the induced representation of $(G, \rho, W)$ in $G L_{n}$. By Weyl's unitarian trick, the theorem is true for complex semi-simple groups. The following proposition follows from Theorem 3.1.

Proposition 3.1. Let $(G, \rho, W)$ be a finite dimensional irreducible representation of a compact subgroup $G$ of $G L_{n}$.

(1) The multiplicity $m\left(\rho \uparrow_{G}^{G L_{n}}(r), \lambda\right)$ of an irreducible representation $\lambda$ of $G L(r)$ in $\rho \uparrow_{G}^{G L_{n}}(r)$ does not depend on $r$.

(2) The irreducible decomposition of $\rho \uparrow{ }_{G}^{G L_{n}}(r)$ contains no irreducible components corresponding to partitions of length $>n$.

\subsection{Basic properties of universal induced characters}

In this section, we will prove some properties of universal induced characters. We use the notation $G L(n)$ instead of $G L_{n}$.

Proposition 3.2.

(1) Let $\lambda$ be a partition of length $\leq n$. Then

$$
\chi(\lambda \uparrow \underset{G L(n)}{G L(n)})=s_{\lambda} .
$$

(2) Let $H \subset G$ be compact subgroups of $G L(n)$. Let $(H, \rho)$ be a finite dimensional irreducible representation of $H$. Then

$$
\chi\left(\rho \uparrow_{H}^{G L(n)}\right)=\chi\left(\left(\rho \uparrow_{H}^{G}\right) \uparrow_{G}^{G L(n)}\right),
$$

where $\rho \uparrow{ }_{H}^{G}$ denotes the (usual) induced representation.

Proof. (1) By the Frobenius reciprocity and Weyl's unitary restriction, we have

$$
\begin{aligned}
\chi\left(\lambda \uparrow_{G L(n)}^{G L L(n)}\right) & =\sum_{\ell(\mu) \leq n} m\left(\lambda \uparrow_{G L(n)}^{G L(n)}, \mu\right) s_{\mu} \\
& =\sum_{\ell(\mu) \leq n} m\left(\mu \downarrow_{G L(n)}^{G L(n)}, \lambda\right) s_{\mu} \\
& =s_{\lambda} .
\end{aligned}
$$

(2) By the Frobenius reciprocity, 


$$
\chi\left(\rho \uparrow_{H}^{G L(n)}\right)=\sum_{\ell(\lambda) \leq n} m\left(\lambda \downarrow_{H}^{G L(n)}, \rho\right) s_{\lambda}
$$

and

$$
\rho \uparrow{ }_{H}^{G}=\sum_{r} m\left(\gamma \downarrow_{H}^{G}, \rho\right) \gamma,
$$

where the sum is over all equivalence classes of irreducible representations $\gamma$ of $G$ and $m\left(\gamma \downarrow_{H}^{G}, \rho\right)$ denotes the multiplicity of $\rho$ in $\gamma \downarrow_{H}^{G}$. By (1), we have

$$
\chi\left(\left(\rho \uparrow_{H}^{G}\right) \uparrow_{G}^{G L(n)}\right)=\sum_{\lambda, r} m\left(\gamma \downarrow_{H}^{G}, \rho\right) m\left(\lambda \downarrow_{G}^{G L(n)}, \gamma\right) s_{\lambda} .
$$

Since

$$
m(\lambda \downarrow \underset{H}{G L(n)}, \rho)=\sum_{r} m\left(\gamma \downarrow_{H}^{G}, \rho\right) m\left(\lambda \downarrow_{G}^{G L(n)} \cdot \gamma\right),
$$

we obtain the desired result.

Proposition 3.3. Let $G_{1}, \cdots, G_{r}$ be compact subgroups of $G L\left(n_{1}\right), \cdots$, $G L\left(n_{r}\right)$, respectively. We consider the product group $G_{1} \times \cdots \times G_{r}$ as a subgroup of $G L\left(n_{1}+\cdots+n_{r}\right)$ by

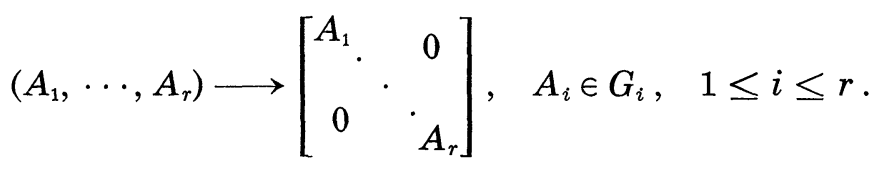

Let $\rho_{1}, \cdots, \rho_{r}$ be finite dimensional representations of $G_{1}, \cdots, G_{r}$, respectively. Then

$$
\left.\chi\left(\rho_{1} \otimes \cdots \otimes \rho_{r} \uparrow_{G_{1} \times \cdots \times G_{r}}^{G L\left(n_{1}+\cdots\right.}+n_{r}\right)\right)=\chi\left(\rho_{1} \uparrow_{G_{1}}^{G L\left(n_{1}\right)}\right) \cdots \chi\left(\rho \uparrow_{G_{r}}^{G L\left(n_{r}\right)}\right) .
$$

Proof. For $m \in N$, we apply the Molien-Weyl formula. Then

$$
\begin{aligned}
& \chi\left(\rho_{1} \otimes \cdots \otimes \rho_{r} \uparrow{ }_{G_{1} \times \cdots \times G_{r}}^{G L\left(n_{1}+\cdots+n_{r}\right)}(m)\right)\left(t_{1}, \cdots, t_{m}\right) \\
& \quad=\prod_{1 \leq i \leq r} \int_{G_{i}} \frac{\operatorname{Tr}\left(\rho_{i}(g)^{-1}\right)}{\prod_{1 \leq j \leq m} \operatorname{det}\left(1_{n_{i}}-g t_{j}\right)} d \mu_{i},
\end{aligned}
$$

where $d \mu_{i}$ is the normalized Haar measure on $G_{i}$

$$
=\prod_{1 \leq i \leq r} \chi\left(\rho_{i} \uparrow \underset{G i}{G L\left(n_{i}\right)}(m)\right)\left(t_{1}, \cdots, t_{m}\right),
$$

which completes the proof.

Proposition 3.4. We consider the product group $G L\left(n_{1}\right) \times \cdots \times$ $G L\left(n_{r}\right)$ as a subgroup of $G L\left(n_{1}+\cdots+n_{r}\right)$. let $\lambda_{1}, \cdots, \lambda_{r}$ be partitions with $\ell\left(\lambda_{1}\right) \leq n_{1}, \cdots, \ell\left(\lambda_{r}\right) \leq n_{r}$, respectively. Then 


$$
\chi\left(\lambda_{1} \otimes \cdots \otimes \lambda_{r} \uparrow \underset{G L\left(n_{1}\right) \times \cdots \times G L\left(n_{r}\right)}{G L\left(n_{1}+\cdots+n_{r}\right)}\right)=s_{\lambda_{1}} \cdots s_{\lambda_{r}}=\sum_{\lambda} L R_{\lambda_{1}, \cdots, \lambda_{r}}^{\lambda} s_{\lambda} .
$$

Proof. This follows from the previous two propositions.

Let $\lambda$ be a partition of length $\leq \sum_{1 \leq i \leq r} n_{i}$. Then by the Frobenius reciprocity theorem, we obtain

$$
m\left(\lambda \downarrow \begin{array}{l}
G L\left(n_{1}+\cdots+n_{r}\right) \\
G L\left(n_{1}\right) \times \cdots \times G L\left(n_{r}\right)
\end{array}, \quad \lambda_{1} \otimes \cdots \otimes \lambda_{r}\right)=L R_{\lambda_{1}, \cdots, \lambda_{r}}^{\lambda},
$$

and hence

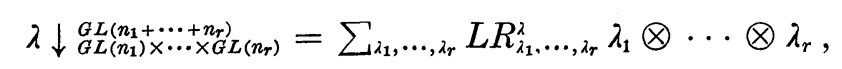

where the sum is over all partitions $\lambda_{1}, \cdots, \lambda_{r}$ with $\ell\left(\lambda_{i}\right) \leq n_{1}, \cdots, \ell\left(\lambda_{r}\right)$ $\leq n_{r}$, respectively.

In particular, putting $n_{1}=n_{2}=\cdots=n_{r}=1$, we obtain a well known formula:

$$
s_{\lambda}=\sum_{\mu} K_{\lambda, \mu} m_{\mu} .
$$

Consider the representation $G L\left(n_{1}\right) \times \cdots \times G L\left(n_{r}\right) \rightarrow G L\left(n_{1} \cdots n_{r}\right)$ defined by $\left(A_{1}, \cdots, A_{r}\right) \rightarrow A_{1} \otimes \cdots \otimes A_{r}, A_{1} \in G L\left(n_{i}\right), 1 \leq i \leq r$. Then we can consider

$$
\begin{aligned}
V\left(n_{1} \cdots n_{r}\right) & :=V\left(n_{1}\right) \otimes \cdots \otimes V\left(n_{r}\right), \\
V\left(n_{1}\right) & :=C^{n_{i}}, \quad 1 \leq i \leq r
\end{aligned}
$$

as a $G L\left(n_{1}\right) \times \cdots \times G L\left(n_{r}\right)$-module.

Proposition 3.5. Let $\lambda_{1}, \cdots, \lambda_{r}$ be partitions of length $\leq n_{1}, \cdots, n_{r}$, respectively and consider the representation $\left(G L\left(n_{1}\right) \times \cdots \times G L\left(n_{r}\right), \lambda_{1} \otimes\right.$ $\left.\cdots \otimes \lambda_{r}\right)$. Then

$$
\begin{aligned}
\left(\lambda_{1} G\right. & \left.\cdots \otimes \lambda_{r} \uparrow \underset{G L\left(n_{1}\right) \times \cdots \times G L\left(n_{r}\right)}{G L\left(n_{1} \cdots n_{r}\right)}\right) \\
& = \begin{cases}s_{\lambda_{1}} * s_{\lambda_{2}} * \cdots * s_{\lambda_{r}}, & \text { if }\left|\lambda_{1}\right|=\cdots=\left|\lambda_{r}\right|, \\
0, & \text { otherwise. }\end{cases}
\end{aligned}
$$

Proof. This follows from Proposition 2.2 and the Molien-Weyl formula.

Let $(G, \rho, W)$ be a finite dimensional representation of a linearly reductive subgroup $G$ of $G L(V), \operatorname{dim}_{C} V=n$. Recall that $\rho \uparrow \uparrow_{G}^{G L(n)}(r), r \in N$, is an $N$-graded module. We denote by $\left[\rho \uparrow_{G}^{G L(n)}(r)\right]$ the subspace with degree $(1, \cdots, 1) \in N^{r}$, that is,

$$
\left[\rho \uparrow_{G}^{G L(n)}(r)\right]=\operatorname{Hom}_{G}\left(V^{\otimes r}, W\right) .
$$


The symmetric group $S_{r}$ acts on $V^{\otimes r}$ by permuting positions:

$$
\sigma\left(v_{1} \otimes \cdots \otimes v_{r}\right)=v_{\sigma-1(1)} \otimes \cdots \otimes v_{\sigma-1(r)} .
$$

On the other hnad, $G$ acts on $V^{\otimes r}$ by

$$
g\left(v_{1} \otimes \cdots \otimes v_{r}\right)=\left(g v_{1}\right) \otimes \cdots \otimes\left(g v_{r}\right) .
$$

Since these two actions of $S_{r}$ and $G$ on $V^{\otimes r}$ commute each other, $\rho \uparrow_{G}^{G L(n)}(r)$ is an $S_{r}$-module. Let $\lambda$ be a partition with $|\lambda|=r$ and denote by $m\left(\left[\rho \uparrow_{G}^{G L(n)}(r)\right],[\lambda]\right)$ the multiplicity of $[\lambda]$ in $\left[\rho \uparrow_{G}^{G L(n)}(r)\right]$.

THEOREM 3.2. Let $G$ be a linearly reductive subgroup of $G L(n)$ and $\rho$ a finite dimensional representation of $G$. For a positive integer $r$, let $\lambda$ be a partition with $|\lambda|=r$. Then, $m\left(\rho \uparrow_{G}^{G L(n)}(r), \lambda\right)=m\left(\left[\rho \uparrow_{G}^{G L(n)}(r)\right],[\lambda]\right)$.

Proof. This follows from Proposition 2.2.

We introduce a multiplication $\circledast$ on $\Lambda$ by

$$
s_{\lambda} \circledast s_{\mu}= \begin{cases}s_{\lambda} * s_{\mu}, & \text { if }|\lambda|=|\mu| \\ 0, & \text { otherwise . }\end{cases}
$$

Proposition 3.6. Let $G_{1}, G_{2}, \cdots, G_{r}$ be compact subgroups of $G L\left(n_{1}\right)$, $G L\left(n_{2}\right), \cdots, G L\left(n_{r}\right)$, respectively. We consider the group $G_{1} \times G_{2} \times \cdots \times G_{r}$ as a subgroup of $G L\left(n_{1} n_{2} \cdots n_{r}\right)$ :

$$
\begin{gathered}
\left(A_{1}, A_{2}, \cdots, A_{r}\right) \longmapsto A_{1} \otimes A_{2} \otimes \cdots \otimes A_{r} \\
\text { with } \quad A_{i} \in G_{i}, \quad 1 \leq i \leq r .
\end{gathered}
$$

Let $\rho_{1}, \rho_{2}, \cdots, \rho_{r}$ be finite dimensional rational representations of $G_{1}$, $G_{2}, \cdots, G_{r}$, respectively. Then

$$
\begin{aligned}
& \chi\left(\rho_{1} \otimes \rho_{2} \otimes \cdots \otimes \rho_{r} \uparrow_{G_{1} G_{2} \cdots G_{1}}^{G L\left(n_{1} n_{2} \cdots n_{r}\right)}\right) \\
& \quad=\chi\left(\rho_{1} \uparrow{ }_{G_{1}}^{G L\left(n_{1}\right)}\right) \circledast \chi\left(\rho_{2} \uparrow_{G_{2}}^{G L\left(n_{2}\right)}\right) \circledast \cdots \circledast \chi\left(\rho_{r} \uparrow \underset{G_{r}}{G L\left(n_{r}\right)}\right) .
\end{aligned}
$$

Proof. This follows from Proposition 3.5.

Consider the adjoint representation Ad: $S L(n) \rightarrow G L\left(n^{2}\right)$ of $S L(n)$. It follows from Proposition 2.1, that

$$
\prod_{i, j, k}\left(1-\varepsilon_{i} \varepsilon_{j}^{-1} t_{k}\right)^{-1}=\sum_{\mu, \nu}\left(s_{\mu} \circledast s_{\nu}\right)(t) s_{\mu}(\varepsilon) s_{\nu}\left(\varepsilon^{-1}\right),
$$

for infinite variables $t=\left(t_{1}, t_{2}, \cdots\right), \varepsilon=\left(\varepsilon_{1}, \varepsilon_{2}, \cdots\right)$.

By the Molien-Weyl formula, we obtain:

TheOREm 3.5. Let $\lambda$ be a partition of length $\leq n$. Then 


$$
\chi\left(\lambda \uparrow_{S L(n)}^{G L(n 2)}\right)=\sum_{\mu, \nu} L_{\lambda \nu}^{\mu}\left(s_{\mu} * s_{\nu}\right),
$$

where the sum is over all partitions $\mu$ and $\nu$ of length $\leq n$. In particular we have

$$
\chi(1 \uparrow \underset{S L(n)}{G L(n 2)})=\sum_{\ell(\lambda) \leq n} s_{\lambda} * s_{\lambda},
$$

Let $C\left[\oplus^{r} M_{n}(C)\right]^{G L(n)}$ be the ring of matrix invariants by the simultaneous adjoint action of $G L(n)$ on the vector space of $r n \times n$ complex matrices $\oplus^{r} M_{n}(C)$. Since

$$
1 \uparrow \underset{S L(n)}{G L\left(n^{2}\right)}(r)=C\left[\oplus^{r} M_{n}(C)\right]^{G L(n)},
$$

we obtain a result of $\mathbf{E}$. Formanek:

TheOREm 3.6 (E. Formanek [F], Theorem 17). The Hilbert series of $C\left[\oplus \oplus^{r} M_{n}(C)\right]^{G L(n)}$ is equal to

$$
\sum s_{\lambda}\left(t_{1}, \cdots, t_{r}\right) * s_{\lambda}\left(t_{1}, \cdots, t_{r}\right),
$$

where the sum is over all partitions of length $\leq n$.

By Theorem 3.5, and Theorem 3.2, we have

$$
\left[1 \uparrow_{S L(n)}^{G L(n 2)}(r)\right]=\bigoplus_{\substack{\ell(\lambda) \leq n \\|\lambda|=r}}([\lambda] \otimes[\lambda]) .
$$

On the other hand,

$$
\begin{gathered}
{\left[1 \uparrow_{S L(n)}^{G L\left(n n^{2}\right)}(r)\right]=\left(\otimes^{r} M_{n}(C)\right)^{G L(n)}} \\
=\text { the } C \text {-vector space of multi-linear } \\
\quad \text { matrix invariants of degree } r .
\end{gathered}
$$

We denote by $I(\lambda)$ the minimal two-sided ideal of the group ring $C\left[S_{r}\right]$ corresponding to a partition $\lambda$ of size $r$. Moreover we put

$$
I(n, r)=\oplus\{I(\lambda)|| \lambda \mid=r, \ell(\lambda) \leq n\} .
$$

Then, as is well known, as $S_{r}$-modules, $I(\lambda)=[\lambda] \otimes[\lambda]$. Hence we obtain a fundamental result on matrix invariants due to Procesi-Razymslov:

Theorem 3.7 (Procesi [P] Theorem 4.3. Razmyslov [R] p. 755).

$$
\left(\otimes^{r} M_{n}(C)\right)^{G L(n)} \cong I(n, r), \quad \text { as } S_{r} \text {-modules . }
$$

In particular, if $r=n$, we obtain Schur's commutator theorem $\operatorname{End}_{G L(n)}\left(V^{\otimes n}\right)=C\left[S_{r}\right]$, where $V=C^{n}$, because $I(n, n)=C\left[S_{n}\right]$ and $\operatorname{End}_{G L(n)}\left(V^{\otimes n}\right)=\left(\otimes^{n} M_{n}(C)\right)^{G L(n)}$. 


\section{§4. Representations of the symplectic group and the orthogonal group}

In this section we shall review some fundamental results on finite dimensional irreducible representations of classical groups $S p(2 n)$, $S O(2 n+1)$ and $S O(2 n)$. For details see [W-2].

In general let $G$ be a connected semi-simple complex Lie group, $K$ a maximal compact subgroup of $G$ and

$$
T=\left\{\left(\varepsilon_{1}, \cdots, \varepsilon_{r}\right)\left|\left(\varepsilon_{1}, \cdots, \varepsilon_{r}\right) \in C^{r},\right| \varepsilon_{i} \mid=1\right\} \quad r=\operatorname{rank} \text { of } T,
$$

a maximal torus of $K$. For each $i, 1 \leq i \leq r$, let $t_{i}$ denote the map $t_{i}$ : $T \rightarrow C, t_{i}\left(\varepsilon_{1}, \cdots, \varepsilon_{r}\right)=\varepsilon_{i}$. Let $\operatorname{Mod}(G)$ denote the Grothendick ring of finite dimensional holomorphic representations of $G$. By the Cartan-Weyl theory, $\operatorname{Mod}(G)$ is determined by the domainant integral weights.

Case $1, G=S p(2 n)$. The symplectic group $\operatorname{Sp}(2 n)$ consists of all linear transformations leaving invariant the non-degenerate skew-symmetric bilinear form

$$
[x, y]=\left(x_{1} y_{1}^{\prime}-x_{1}^{\prime} y_{1}\right)+\cdots+\left(x_{n} y_{n}^{\prime}-x_{n}^{\prime} y_{n}\right)
$$

for

$$
x=\left(x_{1}, \cdots, x_{n}, x_{1}^{\prime}, \cdots, x_{n}^{\prime}\right) \text { and } y=\left(y_{1}, \cdots, y_{n}, y_{1}^{\prime}, \cdots, y_{n}^{\prime}\right) .
$$

We take as $T$ the set of all diagonal matrices

$$
\left(\varepsilon_{\ell}, \cdots, \varepsilon_{n}, \varepsilon_{\ell}^{-1}, \cdots, \varepsilon_{n}^{-1}\right) \text { with } \quad\left\{\varepsilon_{i} \mid=1, \quad 1 \leq i \leq n .\right.
$$

If $M$ is a finite dimensional irreducible $S p(2 n)$-module, the associated dominant integral weight has the form $\omega=\lambda_{1} t_{1}+\cdots+\lambda_{n} t_{n}, \lambda=\left(\lambda_{1}, \cdots\right.$, $\lambda_{n}$ ) is a partition. Therefore the equivalence classes of finite dimensional irreducible $S p(2 n)$-modules correspond bijectively to the set of all partitions of length $\leq n$. If $M$ is a finite dimensional irreducible $S p(2 n)$ module corresponding to a partition $\lambda$ of length $\leq n$, we shall write it by $\rho_{S p(2 n)}(\lambda)$.

Case $2, G=S O(2 n+1)$. The orthogonal group $S O(2 n+1)$ consists of all special linear transformations leaving invariant the non-degenerate symmetric bilinear form

$$
(x, y)=x_{0} y_{0}+\left(x_{1} y_{1}^{\prime}+x_{1}^{\prime} y_{1}\right)+\cdots+\left(x_{n} y_{n}^{\prime}+x_{n}^{\prime} y_{n}\right)
$$

for

$$
x=\left(x_{0}, x_{1}, \cdots, x_{n}, x_{1}^{\prime}, \cdots, x_{n}^{\prime}\right) \quad \text { and } \quad y=\left(y_{0}, y_{1}, \cdots, y_{n}, y_{1}^{\prime}, \cdots, y_{n}^{\prime}\right) .
$$


We take as $T$ the set of all diagonal matrices

$$
\left(1, \varepsilon_{1}, \cdots, \varepsilon_{n}, \varepsilon_{1}^{-1}, \cdots, \varepsilon_{n}^{-1}\right) \quad \text { with } \quad\left|\varepsilon_{i}\right|=1, \quad 1 \leq i \leq n .
$$

If $M$ is a finite dimensional irreducible $S O(2 n+1)$-module, the associated dominant integral weight has the form

$$
\begin{gathered}
\lambda_{1} t_{1}+\cdots+\lambda_{n} t_{n}, \quad \lambda_{1} \geq \lambda_{2} \geq \cdots \geq \lambda_{n-1} \geq \lambda_{n} \geq 0 \\
\text { with } \quad \lambda_{1}, \cdots, \lambda_{n} \in Z .
\end{gathered}
$$

Therefore the equivalence classes of finite dimensional irreducible $S O(2 n+1)$-modules correspond bijectively to the set of all partitions $\lambda$ of length $\leq n$. If $M$ is a finite dimensional irreducible $S O(2 n+1)$ module corresponding to a partition $\lambda$ of length $\leq n$, we shall write it by $\rho_{\text {So(2n+1) }}(\lambda)$.

Case $3, G=S O(2 n)$. The orthogonal group $S O(2 n)$ consists of all special linear transformations leaving invariant the non-degenerate symmetric bilinear form

$$
(x, y)=\left(x_{1} y_{1}^{\prime}+x_{1}^{\prime} y_{1}\right)+\cdots+\left(x_{n} y_{n}^{\prime}+x_{n}^{\prime} y_{n}\right)
$$

for

$$
x=\left(x_{1}, \cdots, x_{n}, x_{1}^{\prime}, \cdots, x_{n}^{\prime}\right) \text { and } y=\left(y_{1}, \cdots, y_{n}, y_{1}^{\prime}, \cdots, y_{n}^{\prime}\right) \text {. }
$$

Take as $T$ the set of all diagonal matrices

$$
\left(\varepsilon_{1}, \cdots, \varepsilon_{n}, \varepsilon_{1}^{-1}, \cdots, \varepsilon_{n}^{-1}\right) \text { with } \quad\left|\varepsilon_{i}\right|=1, \quad 1 \leq i \leq n .
$$

If $M$ is a finite dimensional irreducible $S O(2 n)$-module, the associated dominant weight has the form

$$
\lambda_{1} t_{1}+\cdots+\lambda_{n} t_{n}, \quad \lambda_{1} \geq \lambda_{2} \geq \cdots \lambda_{n-1} \geq\left|\lambda_{n}\right| \quad \text { with } \quad\left\{\lambda_{1}, \cdots, \lambda_{n}\right\} \in Z .
$$

In this case, $\lambda_{n}$ can be negative and irreducible $S O(2 n)$-modules correspond bijectively to the sequences $\lambda=\left(\lambda_{1}, \cdots, \lambda_{n}\right)$ of integers satisfying the condition

$$
\lambda_{1} \geq \lambda_{2} \geq \cdots \geq \lambda_{n-1} \geq\left|\lambda_{n}\right|
$$

For such a sequence $\lambda$, we denote by $\left(\rho_{\lambda}, V_{\lambda}\right)$ the corresponding irreducible representation of $S O(2 n)$ and by $\left(\hat{\rho}, V_{k}\right)$ the representation of $S O(2 n)$ on $V_{\lambda}$ defined by $\hat{\rho}_{\lambda}=\rho_{\lambda} \cdot \omega$, with $\omega=$ the involutive outer automorphism of $S O(2 n)$. 
For a partition $\lambda$ of length $\leq n$, define the representation $\rho_{S o(2 n)}(\lambda)$ of $S O(2 n)$ by

$$
\rho_{S O(2 n)}(\lambda)= \begin{cases}\rho_{\lambda} & \text { if } \lambda_{n}=0, \\ \rho_{\lambda} \oplus \hat{\rho}_{\lambda} & \text { if } \lambda_{n}>0 .\end{cases}
$$

Then $\rho_{S O(2 n)}(\lambda)$ is the representation obtained by an irreducible representation of the orthogonal group $O(2 n)$.

\section{§5. The universal induced characters for the classical groups}

Let $G$ be a classical group of type $B_{n}, C_{n}$ or $D_{n}$. For a partition $\lambda$ of length $\leq n$, let $\rho_{G}(\lambda)$ be the representation of $G$ defined in the previous section. H. Weyl calculated the universal induced character for $\rho_{G}(\lambda)$ in 1926. More recently D.E. Littlewood, R.C. King and K. Koike-I. Terada had shown the restriction formula for $\chi\left(\mu \downarrow_{G}^{G L_{\nu}}\right), \nu=2 n$ if $G=S p(2 n)$ or $S O(2 n) \nu=2 n+1$ if $G=S O(2 n+1)$. In this section we shall rewrite Weyl's formula for the universal induced character and obtain an expression for the universal induced character as an infinite linear combination of Schur functions. By the Frobenius reciprocity theorem, this gives the restriction formula (see [K-1]).

Now we refer to the following theorem.

Theorem $5.1(\mathrm{H}$. Weyl [W-1]). Let $\lambda$ be a partition of length $\leq n$ and $r(>n)$ an integer. Then we have

(1) $\chi\left(\rho_{S p(2 n)}(\lambda) \uparrow_{S p(2 n)}^{G L_{2 n}}(r)\right)$

$$
=\frac{\left|t^{r-1+\lambda_{1}}, t^{r-2+\lambda_{2}}, \cdots, t^{r-n+\lambda_{n}}, t^{r-n-1}, t^{r-n-2}+t^{r-n}, \cdots, 1+t^{2(r-n-1)}\right|}{\prod_{1 \leq i \leq j \leq r}\left(1-t_{i} t_{j}\right)\left|t^{r-1}, t^{r-2}, \cdots, t, 1\right|} .
$$

(2) $\chi\left(\rho_{S O(2 n+1)}(\lambda) \uparrow_{S O(2 n+1)}^{G L_{2 n+1}}(r)\right)$

$$
=\frac{\left|t^{r-1+\lambda_{1}}, t^{r-2+\lambda_{2}}, \cdots, t^{r-n+\lambda_{n}}, t^{r-n-1}+t^{r-n}, \cdots, 1+t^{2(r-n)-1}\right|}{\prod_{1 \leq i \leq j \leq r}\left(1-t_{i} t_{j}\right)\left|t^{r-1}, t^{r-2}, \cdots, t, 1\right|} .
$$

(3) $\chi\left(\rho_{S O(2 n)}(\lambda) \uparrow \underset{S O(2 n)}{G L_{2 n}}(r)\right)$

$$
=\frac{\left|t^{r-1+\lambda_{1}}, t^{r-2+\lambda_{2}}, \cdots, t^{r-n+\lambda_{n}}, t^{r-n-1}-t^{r-n+1}, \cdots, 1-t^{2(r-n)}\right|}{\prod_{1 \leq i \leq j \leq r}\left(1-t_{i} t_{j}\right)\left|t^{r-1}, t^{r-2}, \cdots, t, 1\right|} .
$$

Here in general, for given polynomials $f_{1}(t), \cdots, f_{r}(t), \mid f_{1}(t), f_{2}(t), \cdots$, $f_{r}(t) \mid$ stands for the polynomial in $r$ variables $t_{1}, t_{2}, \cdots, t_{r}$ defined by

$$
\left|f_{1}(t), f_{2}(t), \cdots, f_{r}(t)\right|=\sum_{\sigma \in S_{r}} \operatorname{sgn}(\sigma) f_{\sigma(1)}\left(t_{1}\right) f_{\sigma(2)}\left(t_{2}\right) \cdots f_{\sigma(r)}\left(t_{r}\right) .
$$


The following identities are called Littlewood's identities. For the proof, see [M, p. 45].

LEMMA 5.1. We have the following identities in the ring of symmetric functions in infinitely many variables $t_{1}, t_{2}, \cdots$.

(1) $\sum s_{i}=\prod_{i \leq j}\left(1-t_{i} t_{j}\right)^{-1}$, where the sum is over all even partitions $\lambda$.

(2) $\sum s_{\lambda}=\prod_{i<j}\left(1-t_{i} t_{j}\right)^{-1}$,

where the sum is over all partitions $\lambda$ such that ${ }^{t} \lambda$ are even partitions.

For a Young diagram $\mu$, we mean by a skew-hook $h$ of $\mu$ a connected part of the rim of $\mu$ which can be removed to leave a Young diagram. Let $h$ be a skew-hook of a Young diagram $\mu$ and remove $h$ out of $\mu$. Then we denote by $\mu-h$ the resulting Young diagram. The skew-hook length of $h$ is defined as the number of nodes in $h$ and the leg length $\omega(h)$ of $h$ is defined by

$$
\omega(h)=\max \{j \mid(i, j) \in h\}-\min \{j \mid(i, j) \in h\} .
$$

For a sequence $\left(f_{1}, f_{2}, \cdots, f_{r}\right)$ of $r$ integers, we set

$$
\left\{f_{1}, f_{2}, \cdots, f_{r}\right\}=\left|t^{\ell_{1}}, t^{\ell_{2}}, \cdots, t^{\ell_{r}}\right|,
$$

where $\ell_{i}=f_{i}+r-i$, for $1 \leq i \leq r$. Then we obtain easily the rule:

$$
\left\{\cdots, f_{k}, f_{k+1}, \cdots\right\}=-\left\{\cdots, f_{k+1}-1, f_{k}+1, \cdots\right\} .
$$

In particular if $f_{k+1}=f_{k}+1$ for some $k$, then $\left\{f_{1}, f_{2}, \cdots, f_{r}\right\}=0$.

We begin with a combinatorial

LEMMA 5.2. Let $f=\left(f_{1}, f_{2}, \cdots, f_{r}\right)$ be a sequence of non-negative integers and $f(k)=\left(f_{1}, f_{2}, \cdots, f_{k}\right), k<r, a$ subsequence of $f$. Suppose that $f(k)$ is $a$ partition (i.e. $f_{1} \geq f_{2} \geq \cdots \geq f_{k}$ ).

(1) If there is a partition $g=\left(g_{1}, g_{2}, \cdots, g_{k+1}\right)$ of length $k+1$ satisfying the following two conditions (a) and (b):

(a) $g$ has a skew-hook $h$ of skew-length $f_{k+1}$ through the node $(k+1,1)$,

(b) $f(k)=g-h$, then

$$
\left\{f_{1}, f_{2}, \cdots, f_{r}\right\}=(-1)^{\omega(n)}\left\{g_{1}, g_{2}, \cdots, g_{k+1}, f_{k+2}, \cdots, f_{r}\right\} .
$$

(2) If there is no partition $g$ of length $k+1$ satisfying the two conditions (a) and (b), then

$$
\left\{f_{1}, f_{2}, \cdots, f_{r}\right\}=0 .
$$

Proof. By the rule (*), we obtain, for any $1 \leq j<k$, 
(**) $\quad\left\{f_{1}, \cdots, f_{k}, f_{k+1}, \cdots, f_{r}\right\}$

$$
=(-1)^{j}\left\{f_{1}, f_{2}, \cdots, f_{k-\jmath}, f_{k+1}-j, f_{k-j+1}+1, \cdots, f_{k}+1, f_{k+2}, \cdots, f_{r}\right\} .
$$

The conditions (a) and (b) mean that the sequence

$$
\left(f_{1}, \cdots, f_{k-\omega(h)}, f_{k+1}-\omega(h), f_{k-\omega(h)+1}+1, \cdots, f_{k}+1\right)
$$

is a partition and equal to $g=\left(g_{1}, g_{2}, \cdots, g_{k+1}\right)$. Then (1) follows from $(* *)$.

The assumption of (2) means that

$$
f_{k+1}-j=f_{k-j}+1, \quad \text { for some } j<k .
$$

But then again by $(*)$ and $(* *)$, we obtain $\left\{f_{1}, f_{2}, \cdots, f_{r}\right\}=0$.

ExAMPLE. $f=(4,2,2,5)$ and $f(3)=(4,2,2)$. In this case

$$
g=(4,3,3,3)=\begin{array}{llll}
\times & \times & \times & \times \\
\times & \times & \times & \otimes \\
& \otimes & \otimes & \bigotimes \quad \longleftarrow \text { skew-hook of length } 5
\end{array}
$$

has a skew-hook $h$ of length 5 through the node $(4,1)$. Therefore $\{4,2$, $2,5\}=\{4,3,3,3\}$.

We now describe the universal induced character for the representation $\rho_{G}(\lambda)$ of each classical group $G$.

Case $1, G=S p(2 n)$. In this case the set of irreducible representations of $G$ is parametrized by the set of all partitions of length $\leq n$. For a partition $\lambda$ of length $\leq n$ and a partition $\mu$, we define an integer $\kappa(\mu, \lambda)$ $\in\{-1,0,1\}$ as follows: we divide into two cases,

Case $(\mathrm{a}), \ell(\mu) \leq n+1$.

$$
\kappa(\mu, \lambda):= \begin{cases}1, & \text { if } \mu=\lambda, \\ 0, & \text { otherwise } .\end{cases}
$$

Case (b), $\ell(\mu)>n+1$.

In this case we set $e:=\ell(\mu)-n-1$. If $\mu$ has no skew-hook of length $2 e$ through the node $(\ell(\mu), 1), \kappa(\mu, \lambda)$ is defined to be zero. If $\mu$ has a skewhook $h$ of length $2 e$ through the node $(\ell(\mu), 1)$, we set

$$
\kappa(\mu, \lambda):=(-1)^{\omega(h)} \kappa(\mu-h, \lambda) .
$$

If $\ell(\mu-h)>n+1$, we continue this process. 
ExAmple. $\quad G=S p(8)$,

$$
\begin{aligned}
& \times \times \times \\
& \times \times x \\
& \begin{array}{r}
\times \\
\times
\end{array} \quad \times \times \times
\end{aligned}
$$

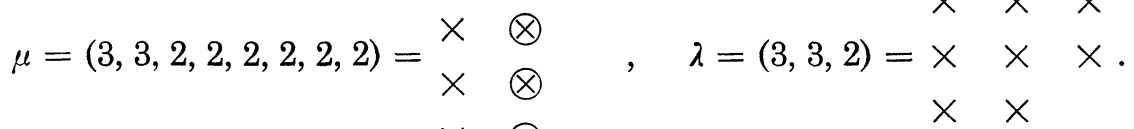

$$
\begin{aligned}
& \times \otimes \\
& \times \otimes \\
& \otimes \otimes
\end{aligned}
$$

There is a skew-hook of length 6 through the node $(8,1)$, and hence $\kappa(\mu, \lambda)=\kappa((3,3,2,1,1,1,1), \lambda)$. The Young diagram

$$
\begin{aligned}
& \times \times \times \\
& \times \times \times \\
& \times \times \\
\mu^{\prime}=(3,3,2,1,1,1,1)= & \otimes
\end{aligned}
$$

has a skew-hook of length 4 through the node $(7,1)$, and hence $\kappa\left(\mu^{\prime}, \lambda\right)$ $=-1$. Thus $k(\mu, \lambda)=-1$.

THEOREM 5.2. Let $\lambda$ be a partition of length $\leq n$. Then

$$
\chi\left(\rho_{S p(2 n)}(\lambda) \uparrow \underset{S p(2 n)}{\left.G L_{2 n}\right)}\right)=\sum_{\nu, \mu, \mu^{\prime}} L R_{\mu, \mu^{\prime}}^{\nu} \kappa(\mu, \lambda) s_{\nu},
$$

where $\nu$ and $\mu$ range over all partitions of length $\leq 2 n$ and $\mu^{\prime}$ ranges over all partitions of length $\leq 2 n$ such that ${ }^{t} \mu^{\prime}$ is an even partition (i.e., with all parts of even numbers).

Proof. By (1) of Theorem 5.1, we have, for any $r>n$,

$$
\begin{aligned}
\text { (*) } \quad \prod_{1 \leq i<j \leq r}\left(1-t_{i} t_{j}\right) \chi\left(\rho_{S p(2 n)}(\lambda) \uparrow \underset{S p(2 n)}{G L_{2 n}}(r)\right) \\
\quad=\sum \frac{\left|t^{r-1+\lambda_{1}}, t^{r-2+\lambda_{2}}, \cdots, t^{r-n+\lambda_{n}}, t^{r-n-1}, t^{\alpha_{1}}, t^{\alpha_{2}}, \cdots, t^{\alpha_{r-n-1}}\right|}{\left|t^{r-1}, t^{r-2}, \cdots, t, 1\right|},
\end{aligned}
$$

where the sum is over all sequences $\left(\alpha_{1}, \alpha_{2}, \cdots, \alpha_{r-n-1}\right)$ of non negative integers such that $\alpha_{i}=r-n-i-1$ or $r-n+i-1$ for all $1 \leq i \leq$ $r-n-1$. 
Let $E$ be the set of all sequences $a=\left(a_{1}, a_{2}, \cdots, a_{r-n-1}\right)$ of non-negative integers such that $a_{i}=0$ or $2 i$ for all $1 \leq i \leq r-n-1$. Then we can rewrite $(*)$ as follows:

$$
\begin{gathered}
\prod_{1 \leq i<j \leq n}\left(1-t_{i} t_{j}\right) \chi\left(\rho_{S p(2 n)}(\lambda) \uparrow \underset{S p(2 n)}{G L_{2 n}}(r)\right)\left|t^{r-1}, t^{r-2}, \cdots, 1\right| \\
=\sum_{a \in E}\left\{\lambda_{1}, \lambda_{2}, \cdots, \lambda, 0, a_{1}, a_{2}, \cdots, a_{r-n-1}\right\} .
\end{gathered}
$$

Let $\mu=\left(\mu_{1}, \mu_{2}, \cdots, \mu_{r}\right), r>n+1$, be a partition and suppose that $\kappa(\mu, \lambda) \neq 0$ and $\ell(\mu)>n+1$. Then $\mu$ has a skew-hook $h$ of length $2(\ell(\mu)$ $-n-1)$ through the node $(\ell(\mu), 1)$. Set $\mu-h=\left(\mu_{1}^{\prime}, \mu_{2}^{\prime}, \cdots, \mu_{\ell(\mu)-1}^{\prime}\right)$. Then this sequence $\mu-h$ is a partition and it follows from (1) of Lemma 5.2 that

$$
(-1)^{\omega(h)}\left\{\mu_{1}, \mu_{2}, \cdots, \mu_{r}\right\}=\left\{\mu_{1}^{\prime}, \mu_{2}^{\prime}, \cdots, \mu_{\ell(\mu)-1}^{\prime}, 0, \cdots, 0\right\} .
$$

Applying this process many times if necessarily, we find that

$$
\kappa(\mu, \lambda)\left\{\mu_{1}, \mu_{2}, \cdots, \mu_{r}\right\}=\left\{\lambda_{1}, \lambda_{2}, \cdots, \lambda_{n}, 0, a_{1}, a_{2}, \cdots, a_{r-n-1}\right\}
$$

for some $\left(a_{1}, a_{2}, \cdots, a_{r-n-1}\right) \in E$.

Conversely take a sequence $a=\left(a_{1}, a_{2}, \cdots, a_{r-n-1}\right) \in E$ and suppose that $\left\{\lambda_{1}, \lambda_{2}, \cdots, \lambda_{n}, 0, a_{1}, a_{2}, \cdots, a_{r-n-1}\right\}=0$. If $a \neq 0$, let $a_{m}$ be the first non zero number in the sequence $\left(a_{1}, a_{2}, \cdots, a_{r-n-1}\right)$. Then by (2) of Lemma 5.2, there is a partition $g=\left(g_{1}, g_{2}, \cdots\right)$ of length $n+m+1$ such that $g$ has a skew-hook $h$ of length $2 m$ through the node $(n+m+1,1)$ satisfying $\left(\lambda_{1}, \lambda_{2}, \cdots, \lambda_{n}\right)=g-h$. Then by (1) of Lemma 5.2, we see that

$$
\begin{aligned}
& \left\{\lambda_{1}, \lambda_{2}, \cdots, \lambda_{n}, 0, a_{1}, a_{2}, \cdots, a_{r-n-1}\right\} \\
& \quad=(-1)^{\omega(h)}\left\{g_{1}, g_{2}, \cdots, g_{n+m+1}, a_{m+1}, a_{m+2}, \cdots, a_{r-n-1}\right\} .
\end{aligned}
$$

Repeating this process, we can construct a partition $\mu$ satisfying the equation $(* *)$ with $\kappa(\mu, \lambda) \neq 0$. Therefore we obtain

$$
\prod_{1 \leq i<j \leq n}\left(1-t_{i} t_{j}\right) \chi\left(\rho_{S p(2 n)}(\lambda) \uparrow_{S p(2 n)}^{G L_{2 n}}(r)\right)=\sum_{\ell(\mu) \leq r} k(\mu, \lambda) s_{\mu}\left(t_{1}, \cdots, t_{r}\right) .
$$

Using Littlewood's formula ((2) of Lemma 5.1) and Proposition 3.1, this yields the desired result.

Proposition 5.1 (The restriction rule from $G L_{2 n}$ to $S p(2 n)$ ).

Let $\nu$ be a partition of length $\leq 2 n$ and $\lambda$ a partition of length $\leq n$. Then

$$
m\left(\nu \uparrow \underset{S p(2 n)}{G L_{2 n}}, \rho_{S p(2 n)}(\lambda)\right)=\sum_{\mu, \mu^{\prime}} \kappa(\mu, \lambda) L R_{\mu^{\prime}, \mu}^{\nu}
$$


where $\mu$ and $\mu^{\prime}$ range over all partitions of length $\leq 2 n$ such that ${ }^{t} \mu^{\prime}$ is an even partition.

Proof. This is an immediate consequence from the Frobenius reciprocity and Theorem 5.2.

Case $2, G=S O(2 n+1)$. In this case the set of irreducible representations of $G$ is parametrized by the set of all partitions of length $\leq n$. For a partition $\lambda$ of length $\leq n$ and a partition $\mu$, we define an integer $\kappa(\mu, \lambda) \in\{-1,0,1\}$ as follows: we divide into two cases,

Case (a), $\ell(\mu) \leq n . \quad$ In this case

$$
\kappa(\mu, \lambda):= \begin{cases}1, & \text { if } \mu=\lambda, \\ 0, & \text { otherwise }\end{cases}
$$

Case (b), $\ell(\mu)>n$. In this case we set $e:=\ell(\mu)-n$. If $\mu$ has no skew-hook of length $2 e-1$ through the node $(\ell(\mu), 1), \kappa(\mu, \lambda)$ is defined to be zero. If $\mu$ has a skew-hook $h$ of length $2 e-1$ through the node $(\ell(\mu), 1)$, we set

$$
\kappa(\mu, \lambda):=(-1)^{\omega(h)} \kappa(\mu-h, \lambda) .
$$

If $\ell(\mu-h)>n$, we repeat this process.

ExAMPLE. $G=S O(7)$,

$$
\begin{aligned}
& \times \times \times \times \times \\
& \times \times \times \times \\
& \times \times \otimes \\
& \times \times(5,4,3,3,3,3,2)= \\
& \times \times \otimes \\
& \times \otimes \otimes \\
& \otimes \otimes
\end{aligned}
$$

and

$$
\begin{aligned}
& x \times \times \times x \\
& \lambda=(5,4,1)=\underset{\times}{\times} \times \times \times
\end{aligned}
$$

In this case $\mu$ has a skew-hook of length 7 through the node $(7,1)$ and hence $\kappa(\mu, \lambda)=\kappa\left(\mu^{\prime}, \lambda\right)$, where 


$$
\mu^{\prime}=\begin{array}{lllll}
\times & \times & \times & \times & \times \\
\times & \times & \times & \times & \\
\times & \otimes & & & \\
\times & \otimes & & & \\
\otimes & \otimes & & \\
\otimes & & &
\end{array}
$$

Since $\mu$ has a skew-hook of length 5 through the node $(6,1)$, we have $\kappa\left(\mu^{\prime}, \lambda\right)=-\kappa\left(\mu^{\prime \prime}, \lambda\right)$, where

$$
\begin{aligned}
& x \times \times \times \times \\
& \mu^{\prime \prime}=\underset{\times}{ } \underset{\times}{ } \times
\end{aligned}
$$

The Young diagram $\mu^{\prime \prime}$ has a skew-hook of length 1 through the node $(4,1)$, and hence $\kappa\left(\mu^{\prime \prime}, \lambda\right)=1$. Thus $\kappa(\mu, \lambda)=-1$.

TheOREm 5.3. Let $\lambda$ be a partition of length $\leq n$. Then

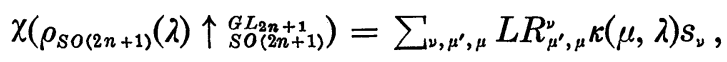

where $\nu$ and $\mu$ range over all partitions of length $\leq 2 n$ and $\mu^{\prime}$ ranges over all partitions of length $\leq 2 n$ such that $\mu$ is an even partition.

Proof. By (2) of Theorem 5.1, we have, for any $r>n$,

$$
\begin{aligned}
& \prod_{1 \leq i \leq j \leq r}\left(1-t_{i} t_{j}\right) \chi\left(\rho_{S O(2 n+1)} \lambda \uparrow \underset{\substack{G L_{2 n+1} \\
S O(2 n+1)}}{ }(r)\right) \\
& \sum \frac{\left|t^{r-1+\lambda_{1}}, t^{r-2+\lambda_{2}}, \cdots, t^{r-n+\lambda_{n}}, t^{\alpha_{1}}, t^{\alpha_{2}}, \cdots, t^{\alpha_{r-n}}\right|}{\left|t^{r-1}, t^{r-2}, \cdots, t, 1\right|}
\end{aligned}
$$

where the sum is over all sequences $\left(\alpha_{1}, \alpha_{2}, \cdots, \alpha_{r-n}\right)$ of non-negative integers such that $\alpha_{i}=r-n-i$ or $r-n+i-1$ for all $1 \leq i \leq r-n$.

Let $E$ be the set of all sequences $a=\left(a_{1}, a_{2}, \cdots, a_{r-n}\right)$ of non-negative integers such that $a_{i}=0$ or $2 i-1$, for all $1 \leq i \leq r-n$. Then we have

$$
\begin{aligned}
& \prod_{1 \leq i \leq j \leq r}\left(1-t_{i} t_{j}\right) \chi\left(\rho_{S O(2 n+1)}(\lambda) \uparrow_{\substack{G L_{2 n+1} \\
S O(2 n+1)}}(r)\right)\left|t^{r-1}, t^{r-2}, \cdots, 1\right| \\
& \quad=\sum_{a \in E}\left\{\lambda_{1}, \lambda_{2}, \cdots, \lambda, a_{1}, a_{2}, \cdots, a_{r-n}\right\} .
\end{aligned}
$$

One may proceed in a fashion analogous to the case of the symplectic group $S p(2 n)$ and obtain the equation

$$
\prod_{1 \leq i \leq j \leq n}\left(1-t_{i} t_{j}\right) \chi\left(\rho_{S O(2 n+1)}(\lambda) \uparrow_{S O(2 n+1)}^{G L_{2 n+1}}(r)\right)=\sum_{\ell(\mu) \leq r} \kappa(\mu, \lambda) s_{\mu}\left(t_{1}, \cdots, t_{r}\right) .
$$


Using Littlewood's formula ((1) of Lemma 5.1) and Proposition 3.1, we obtain the desired result.

By the Frobenius reciprocity theorem, we have the following

Proposition 5.2 (The restriction rule from $G L_{2 n+1}$ to $S O(2 n+1)$ ).

Let $\nu$ be a partition of length $\leq 2 n+1$ and $\lambda$ a partition of length $\leq n$. Then

$$
m\left(\nu \uparrow \underset{S O(2 n+1)}{G L_{2 n+1}}, \rho_{S O(2+1)}(\lambda)\right)=\sum_{\mu, \mu^{\prime}} k(\mu, \lambda) L R_{\mu, \mu^{\prime}}^{\nu},
$$

where $\mu$ ranges over all partitions of length $\leq 2 n+1$ and $\mu^{\prime}$ ranges over all even partitions of length $\leq 2 n+1$.

Case $3, G=S O(2 n)$. In this case the set of representations of $G$ obtained from the restriction of irreducible representations of $O(2 n)$ is parametrized by the set of all partitions of length $\leq n$. For a partition $\lambda$ of length $\leq n$ and a partition $\mu$, we define an integer $\kappa(\mu, \lambda) \in\{-1,0,1\}$ as follows:

Case $(\mathrm{a}), \ell(\mu) \leq n$. In this case we set

$$
\kappa(\mu, \lambda)= \begin{cases}1, & \text { if } \mu=\lambda, \\ 0, & \text { otherwise } .\end{cases}
$$

Case (b), $\ell(\mu)>n$. In this case we set $e:=\ell(\mu)-n$. If $\mu$ has no skew-hook of length $2 e$ through the node $(\ell(\mu), 1)$, we set $\kappa(\mu, \lambda)=0$. If has a skew-hook $h$ of length $2 e$ through the node $(\ell(\mu), 1)$, we set

$$
\kappa(\mu, \lambda):=(-1)^{\omega(h)+1} \kappa(\mu-h, \lambda) .
$$

If $\kappa(\mu-h)>n$, we repeat this process.

ExAMPLE. $G=S O(6)$,

$$
\begin{aligned}
& x \times \times \times x \\
& x \times \times \times \\
& \mu=(5,4,3,3,3,2)=\begin{array}{lll}
\times & \times & \otimes \\
\times & \times & \otimes
\end{array} \quad \text { and } \lambda=(5,4,1) . \\
& \times \otimes \otimes \\
& \otimes \otimes
\end{aligned}
$$

The Young diagram $\mu$ has a skew-hook of length 6 through the node $(6,1)$. Hence $\kappa(\mu, \lambda)=\kappa\left(\mu^{\prime}, \lambda\right)$, where 


$$
\begin{aligned}
& x \times \times \times \times \\
& x \times x \times \\
& \mu^{\prime}=\times \otimes \\
& \otimes \otimes \\
& \otimes
\end{aligned}
$$

The Young diagram $\mu^{\prime}$ has a skew-hook of length 4 through the node $(5,1)$, and hence we have $\kappa\left(\mu^{\prime}, \lambda\right)=-\kappa(\lambda, \lambda)=-1$. Thus $\kappa(\mu, \lambda)=-1$.

THEOREM 5.4. Let $\lambda$ be a partition of length $\leq n$. Then

$$
\chi\left(\rho_{S O(2 n)}(\lambda) \uparrow \underset{S O(2 n)}{G L_{2 n}}\right)=\sum_{\nu, \mu, \mu^{\prime}} L R_{\mu^{\prime}, \mu}^{\nu} k(\mu, \lambda) s_{\nu},
$$

where $\nu$ and $\mu$ range over all partitions of length $\leq 2 n$ and $\mu$ ranges over all partitions of length $\leq 2 n$ such that $\mu$ is an even partition.

Proof. By (3) of Theorem 5.1, for any $r>n$,

$$
\begin{aligned}
& \prod_{1 \leq i \leq j \leq r}\left(1-t_{i} t_{j}\right) \chi\left(\rho_{S O(2 n)}(\lambda) \uparrow_{S}^{G L_{2 n}(2 n)}(r)\right) \\
& =\sum \frac{\left|t^{r-1+\lambda_{1}}, t^{r-2+\lambda_{2}}, \cdots, t^{r-n+\lambda_{n}}, \varepsilon_{1} t^{\alpha_{1}}, \varepsilon_{2} t^{\alpha_{2}}, \cdots, \varepsilon_{r-n} t^{\alpha_{r-n}}\right|}{\left|t^{r-1}, t^{r-2}, \cdots, t, 1\right|},
\end{aligned}
$$

where the sum is over all sequences $\left(\alpha_{1}, \alpha_{2}, \cdots, \alpha_{r-n}\right)$ of non-negative integers such that $\alpha_{i}=r-n-i$ or $r-n+i$, for all $1 \leq i \leq r-n$, and

$$
\varepsilon_{i}=\left\{\begin{aligned}
1, & \text { if } \alpha_{i}=r-n-i, \\
-1, & \text { if } \alpha_{i}=r-n-i .
\end{aligned}\right.
$$

Then the rest of the proof goes in the same way as Theorem 5.2.

By the Frobenius reciprocity theorem, we obtain

Proposition 5.3 (The restriction rule from $G L_{2 n}$ to $S O(2 n)$ ).

Let $\nu$ be a partition of length $\leq 2 n$ and $\lambda$ a partition of length $\leq n$. Then

$$
m\left(\nu \downarrow{ }_{S O(2 n))}^{G L_{2 n}}, \rho_{S O(2 n)}(\lambda)\right)=\sum_{\mu, \mu^{\prime}} k(\mu, \lambda) L R_{\mu^{\prime}, \mu}^{\nu},
$$

where $\mu$ ranges over all partitions of length $\leq 2 n$ and $\mu^{\prime}$ ranges over all even partitions of length $\leq 2 n$.

For a pratition $\lambda=\left(\lambda_{1}, \lambda_{2}, \cdots, \lambda_{n}\right)$ with $\lambda_{n}>0$, consider the irreducible representations $\left(\rho_{\lambda}, V_{\lambda}\right)$ and $\left(\hat{\rho}_{\lambda}, V_{\lambda}\right)$ of $S O(2 n)$, (see $\left.\S 4\right)$. The following result is obtained by the method similar to that used in the proof of Theorem 5.1, (cf. [W-2], p. 229). 
Proposition 5.4. If $r>n$, we have

(1) $\chi\left(\rho_{\lambda} \uparrow \underset{S O(2 n)}{G L_{22}}(r)\right)=\frac{1}{2}\left\{\chi\left(\rho_{S O(2 n)}(\lambda) \uparrow_{S O(2 n)}^{G L_{2 n}}(r)\right)+\hat{\chi}\left(\rho_{S O(2 n)}(\lambda) \uparrow_{S O(2 n)}^{G L_{2 n}}(r)\right)\right\}$,

(2) $\chi\left(\hat{\rho}_{\lambda} \uparrow \underset{S O(2 n)}{G L_{22}}(r)\right)=\frac{1}{2}\left\{\chi\left(\rho_{S O(2 n)}(\lambda) \uparrow \underset{S O(2 n)}{G L_{2 n}}(r)\right)-\hat{\chi}\left(\rho_{S O(2 n)}(\lambda) \uparrow \underset{S O(2 n)}{G L_{2 n}}(r)\right)\right\}$, where

$$
\begin{aligned}
& \hat{\chi}\left(\rho_{S O(2 n)}(\lambda) \uparrow_{\substack{G L_{2 n} \\
S O(2 n)}}(r)\right) \\
& \quad=\frac{\left|t^{r-1+\lambda_{1}}, t^{r-2+\lambda_{2}}, \cdots, t^{r-n+\lambda_{n}}, t^{r-n-1}+t^{r-n+1}, \cdots, 1+t^{2(r-n)}\right|}{\prod_{1 \leq i \leq j \leq r}\left(1-t_{i} t_{j}\right)\left|t^{r-1}, t^{r-2}, \cdots, t, 1\right|} .
\end{aligned}
$$

We set

$$
\hat{\chi}\left(\rho_{S O(2 n)}(\lambda) \uparrow_{S O(2 n)}^{G L_{2 n}}\right)=\varliminf_{\lim } \hat{\chi}\left(\rho_{S O(2 n)}(\lambda) \uparrow_{S O(2 n)}^{G L_{2 n}}(r)\right) .
$$

Then we obtain the following theorem.

TheOREM 5.5. Let $\lambda=\left(\lambda_{1}, \lambda_{2}, \cdots, \lambda_{n}\right), \lambda_{n}>0$, be a partition of length n. Then

$$
\hat{\chi}\left(\rho_{S O(2 n)}(\lambda) \uparrow \underset{S O(2 n)}{G L_{2 n}}\right)=\sum_{\nu, \mu, \mu^{\prime}} L R_{\mu, \mu^{\prime}}^{\nu} \kappa(\mu, \lambda) s_{\nu},
$$

where $\nu$ and $\mu$ range over all partitions of length $\leq 2 n$ and $\mu^{\prime}$ ranges over all even partitions of length $\leq 2 n$. Here the integer $\kappa(\mu, \lambda)$ is defined as follows: If $\ell(\mu) \leq n, \kappa(\mu, \lambda)$ is 1 or 0 , according as $\mu=\lambda$ or $\mu \neq \lambda$. If $\ell(\mu)>n$, let $e:=\ell(\mu)-n$, If $\mu$ has no skew-hook of length $2 e$ through the node $(\ell(\mu), 1)$, then $\kappa(\mu, \lambda)$ is zero. If $\mu$ has a skew-hook of length $2 e$ through the node $(\ell(\mu), 1)$, then

$$
\kappa(\mu, \lambda)=(-1)^{\omega(h)} \kappa(\mu-h, \lambda) .
$$

The universal induced characters for the representations $\rho_{\lambda}$ and $\hat{\rho}_{\lambda}$ are obtained from the equations below.

$$
\chi\left(\rho_{\lambda} \uparrow \underset{S O(2 n)}{G L_{2 n}}\right)=\frac{1}{2}\left\{\chi\left(\rho_{S O(2 n)}(\lambda) \uparrow \underset{S O(2 n)}{G L_{2 n}}\right)+\hat{\chi}\left(\rho_{S O(2 n)}(\lambda) \uparrow \underset{S O(2 n)}{G L_{22}}\right)\right\}
$$

and

$$
\chi\left(\hat{\rho}_{\lambda} \uparrow \underset{S O(2 n)}{G L_{2 n}}\right)=\frac{1}{2}\left\{\chi\left(\rho_{S O(2 n)}(\lambda) \uparrow_{S O(2 n)}^{G L_{2 n}}\right)-\hat{\chi}\left(\rho_{S O(2 n)}(\lambda) \uparrow_{S O(2 n)}^{G L_{2 n}}\right)\right\}
$$

We give a version of the Frobenius reciprocity theorem.

Let $G$ be a complex semi-simple subgroup of $G L_{n}$ and consider the character function $\chi_{G}(\rho)\left(t_{1}, t_{2}, \cdots, t_{r}\right), r=\operatorname{rank}$ of $G$, as a polynomial in 
$t_{1}, t_{1}^{-1}, \cdots, t_{r}, t_{r}^{-1}$. Let $R=C\left[t_{1}, t_{1}^{-1}, \cdots, t_{r}, t_{r}^{-1}\right]$ be the Laurent polynomial ring in the variables $t_{1}, t_{2}, \cdots, t_{r}$ and $R \llbracket z_{1}, z_{2}, \cdots \rrbracket$ the formal power series ring of infinite independent variables $z_{1}, z_{2}, \cdots$ over $R$.

Proposition 5.5. In the formal power series ring $R \llbracket z_{1}, z_{2}, \cdots \rrbracket$, the following formulas fold:

$$
\begin{aligned}
& \sum_{\rho} \chi_{G}(\rho)\left(t_{1}, t_{2}, \cdots, t_{r}\right) \chi\left(\rho \uparrow_{G}^{G L_{n}}\right)\left(z_{1}, z_{2}, \cdots\right) \\
& \quad=\sum_{\lambda} \chi\left(\lambda \downarrow_{G}^{G L_{n}}\right)\left(t_{1}, t_{2}, \cdots, t_{r}\right) s_{\lambda}\left(z_{1}, z_{2}, \cdots\right),
\end{aligned}
$$

where $\rho$ ranges over all equivalence classes of finite dimensional holomorphic representations of $G$ and $\lambda$ ranges over all partitions of length $\leq n$.

Proof. This follows from the Frobenius reciprocity theorem and the orthogonal relation of irreducible characters.

Putting $z_{n+1}=z_{n+2}=\cdots=0$, consider the formal power series ring $R \llbracket z_{1}, z_{2}, \cdots, z_{n} \rrbracket$ over the ring $R$.

Proposition 5.6 (Littlewood's formulas, cf. [L] or [K-T], p. 486). In the ring $R \llbracket z_{1}, z_{2}, \cdots, z_{n} \rrbracket$, the following formulas hold:

$$
\begin{gathered}
\text { (1) } \sum_{2} \frac{\chi_{S p(2 n)}\left(\rho_{S p(2 n)}(\lambda)\right)\left(t_{1}, t_{2}, \cdots, t_{n}\right) s_{\lambda}\left(z_{1}, z_{2}, \cdots, z_{n}\right)}{\prod_{1 \leq i \leq j \leq n}\left(1-t_{i} t_{j}\right)} \\
=\sum_{\lambda} s_{\lambda}\left(t_{1}, \cdots, t_{n}, t_{1}^{-1}, \cdots, t_{n}^{-1}\right) s_{\lambda}\left(z_{1}, z_{2}, \cdots, z_{n}\right) . \\
\text { (2) } \sum_{2} \frac{\chi_{S O(2 n+1)}\left(\rho_{S O(2 n+1)}(\lambda)\right)\left(t_{1}, t_{2}, \cdots, t_{n}\right) s_{\lambda}\left(z_{1}, z_{2}, \cdots, z_{n}\right)}{\prod_{1 \leq i \leq j \leq n}\left(1-t_{i} t_{j}\right)} \\
=\sum_{\lambda} s_{\lambda}\left(1, t_{1}, \cdots, t_{n}, t_{1}^{-1}, \cdots, t_{n}^{-1}\right) s_{\lambda}\left(z_{1}, z_{2}, \cdots, z_{n}\right) . \\
\text { (3) } \sum_{\lambda} \frac{\chi_{S O(2 n)}\left(\rho_{S O(2 n)}(\lambda)\right)\left(t_{1}, t_{2}, \cdots, t_{n}\right) s_{\lambda}\left(z_{1}, z_{2}, \cdots, z_{n}\right)}{\prod_{1 \leq i \leq j \leq n}\left(1-t_{i} t_{j}\right)} \\
=\sum_{\lambda} s_{\lambda}\left(t_{1}, \cdots, t_{n}, t_{1}^{-1}, \cdots, t_{n}^{-1}\right) s_{\lambda}\left(z_{1}, z_{2}, \cdots, z_{n}\right) .
\end{gathered}
$$

Here $\lambda$ ranges over all partitions of length $\leq n$.

Proof. This follows from Proposition 5.5 and Weyl's formulas (Theorem 5.1).

\section{REFERENCES}

[F] E. Formanek, Invariants and the ring of generic matrices, J. Algebra, 89 (1984), 178-223.

[G] J. A. Green, "Polynomial Representations of $G L_{n}$," Lecture Notes in Mathematics No. 830, Springer Verlag, Berlin/Heidelberg/New York, 1980.

[H-R] M. Hochster and J. L. Roberts, Rings of invariants of reductive groups acting on regular rings are Cohen-Macaulay, Adv, in Math., 13 (1974), 115-175. 
[K-1] R. C. King, Modification rules and products of irreducible representations of unitary, orthogonal, and symplectic groups, J. Math. Phys., 12 (1971), 1588-1598.

[K-2] - Branching rules for classical Lie groups using tensor and spinor methods, J. Phys., A 8 (1975), 429-449.

[K-T] K. Koike and I. Terada, Young diagrammatic methods for representation theory of the classical groups of type $\mathrm{B}_{n}, \mathrm{C}_{n}, \mathrm{D}_{n}$, J. Algebra, 107 (1987), 466-511.

[L] D. E. Littlewood, "The Theory of Group Characters and Matrix Representations of Groups," Oxford Univ. Press, London, 1950.

[M] I. G. Macdonald, "Symmetric Functions and Hall Polynomials," Oxford Univ. Press, Oxford, 1979.

[P] C. Procesi, The invariant theory of $n \times n$ matrices, Adv. in Math., 19 (1976), 306381.

[R] Y. P. Razmyslov, Trace identities of full matrix algebras over a field of characteristic zero, Izv. Akad. Nauk SSSR Ser. Mat. 38 (1974), 723-756 (Russian); Math. USSR Izv. 8 (1974), 727-760 (English Translation).

[S] I. Schur, Über eine Klasse von Matrizen die sich einer gegebenen Matrix zuordonen lassen, Dissertation 1901, Berlin. (= Ges. Abhandlungen Bd I, 1-72).

[S] R. Stanley, Invariants of finite groups and their applications to combinatorics, Bull. Amer. Math. Soc., 1 (1979), 475-511.

[T] Y. Teranishi, The Hilbert series of rings of matrix concomitants, Nagoya Math. J., 111 (1988), 143-156.

[W-1] H. Weyl, Zur Darstellungstheorie und Invariantenabzälung der projectiven, der Komplex- und der Drehungsgruppe, Ges. Abhandlungen Bd III, p. 1-25, SpringerVerlag, Berlin/Heidelberg/New York, 1968.

[W-2] —, "The Classical Groups," Princeton Univ. Press, Princeton, N.J., 1946.

Universität Mannheim

Lehrstuhl für Mathematik VI

6800 Mannheim 1,

Federal Republic of Germany

and

Department of Mathematics

Faculty of Science

Nagoya University

Chikusa-ku, Nagoya, 464

Japan 
